\title{
High Mass Star Formation. III. The Functional Form of the Submillimeter Clump Mass Function
}

\author{
Michael A. Reid and Christine D. Wilson \\ Department of Physics and Astronomy, McMaster University, Hamilton, ON, L8S 4M1, \\ Canada \& Harvard-Smithsonian Submillimeter Array Project, $645 \mathrm{~N}$ A'ohoku Pl., Hilo, HI, \\ 96720, USA
}

\begin{abstract}
We investigate the mass function of cold, dusty clumps in 11 low- and highmass star-forming regions. Using a homogeneous fitting technique, we analyze the shape of each region's clump mass function and examine the commonalities among them. We find that the submillimeter continuum clump mass function in low-mass star-forming regions is typically best fit by a lognormal distribution, while that in high-mass star-forming regions is better fit by a double power law. A single power law clump mass distribution is ruled out in all cases. Fitting all of the regions with a double power law, we find the mean power law exponent at the high-mass end of each mass function is $\alpha_{\text {high }}=-2.4 \pm 0.1$, consistent with the Salpeter result of $\alpha=-2.35$. We find no region-to-region trend in $\alpha_{\text {high }}$ with the mass scale of the clumps in a given region, as characterized by their median mass. Similarly, non non-parametric tests show that the shape of the clump mass function does not change much from region to region, despite the obvious changes in the intrinsic mass scale. This result is consistent with the hypothesis that the clump mass distribution is determined by a highly stochastic process, such as turbulent fragmentation. It may also suggest that the data reduction and analysis techniques strongly affect the shape of the derived mass function.
\end{abstract}

Subject headings: stars: formation — submillimeter — ISM: structure — methods: data analysis

\section{INTRODUCTION}

Advances in submillimeter continuum imaging techniques in the past decade have dramatically improved our understanding of the structure of the dense, cold interstellar medium (ISM) in star-forming regions. In every region studied, the cold, dusty ISM exhibits a clumpy, 
often filamentary structure whose density maxima correspond to sites of present or future star formation activity. The primary metric used to describe this inhomogeneous structure is the clump/core ${ }^{1}$ mass function. Submillimeter continuum observations of the core mass function in nearby regions of low-mass star formation, such as Serpens (Testi \& Sargent 1998), $\rho$ Oph (Motte, André, \& Neri 1998; Johnstone et al. 2000), and Orion B (Motte et al. 2001; Johnstone et al. 2001), have provided compelling evidence that the mass function of cold, dense cores mirrors the stellar initial mass function (IMF, e.g. Kroupa 2002, Chabrier 2003). It appears, therefore, that these dusty cores are the immediate precursors of stars. Two main arguments are typically advanced in favor of this interpretation. First, both the stellar and core mass functions are well fit by Salpeter-like power laws (Salpeter 1955) above $\sim 1 M_{\odot}$. Second, both the core and stellar mass functions appear to flatten out below $\sim 1 M_{\odot}$ and peak at about $0.1 M_{\odot}$ (Testi \& Sargent 1998; Motte, André, \& Neri 1998; Johnstone et al. 2000, 2001; Motte et al. 2001; Kroupa 2002). In other words, the core mass function and the stellar IMF have similar shapes and intrinsic mass scales. Thus, by applying a more or less constant core-to-star mass conversion efficiency, the core mass function can be converted into the stellar IMF.

In papers I and II in this series, we showed that, despite the large difference in the median clump mass, the clump mass function in massive star-forming regions NGC 7538 and M17 has a similar shape to that in low-mass star-forming regions (Reid \& Wilson 2005, 2006; Papers I and II hereafter). In both NGC 7538 and M17, we found that double power law fits to the clump mass functions produce exponents which are consistent with the Salpeter mass function (Salpeter 1955). The similarities in their shapes suggests that the clump mass functions in low- and high-mass star-forming regions may have similar origins. What if the mass function of massive clumps were found to resemble that of massive stars, as is the case for low-mass stars? That would suggest that massive clumps could also be converted to massive stars (or small multiple systems) on a more or less one-to-one basis. Such a finding would lend weight to theories which suggest that massive stars form by the collapse of individual clumps (McKee \& Tan 2002, 2003; Krumholz, McKee, \& Klein 2005; Krumholz 2006), rather than those which suggest that massive stars form by coalescence or competitive accretion (Bonnell et al. 1997, 2001; Bonnell, Vine, \& Bate 2004; Bonnell, Larson, \& Zinnecker 2006).

\footnotetext{
${ }^{1}$ Throughout this paper, we reserve the term "core" to denote the small (diameter $\lesssim 0.1 \mathrm{pc}$ ), dense condensations thought to be the immediate precursors of individual stars or small multiple systems. We use the term "clump" more generally to denote discrete structures which may form zero, one, or many stars and may therefore be much more massive than "cores". In this sense, cores may be considered a subset of clumps.
} 
To address these important matters, it is necessary to be able to accurately fit and interpret clump mass functions. In Paper I, we argued that variations in analytic technique might account for much of the observed variation in the power-law exponents derived from fits to submillimeter continuum clump mass functions. In particular, we suggested that the systematic differences between the power-law exponents of clump mass functions in low- and high-mass regions probably result from the fact that the former are typically fit with two power law segments, while the latter are usually fit with only one (compare Motte et al. (2001) and Johnstone et al. (2000) with Tothill et al. (2002) and Mookerjea et al. (2004)). In this paper, we extend and develop these arguments using a more thorough study of the available data on the submillimeter continuum clump mass function. In $\S 2$, we discuss how best to fit a clump/core mass function, paying special attention to means by which the fits may be misinterpreted. In $\S 3$, we apply a consistent fitting methodology to determine which of several plausible functions best represents the clump/core mass function in a variety of star-forming regions. Finally, in $\S 4$, we use non-parametric tests to determine whether the shape of the clump/core mass function varies significantly from region to region or changes with the mass scale studied.

\section{FITTING AND INTERPRETING CLUMP MASS FUNCTIONS}

Clump mass functions are commonly expressed in two forms: differential and cumulative. The differential mass function (DMF), $\Delta N / \Delta M$, has the advantages of permitting a simple Poisson uncertainty analysis but suffers the arbitrariness of binning. Binning can be a serious impediment to the accurate interpretation of stellar mass functions (Scalo 1998; Maíz-Apellániz \& Úbeda 2005). Thus, the differential mass function is not suitable for use when the total number of clumps, $N_{\mathrm{cl}}$, is small. The cumulative mass function (CMF), $N(>M)$, requires a more complicated uncertainty analysis, but it suffers none of the problems associated with binning and therefore is suitable even in the small $N_{\mathrm{cl}}$ regime. The CMF is therefore preferable for studies of the submillimeter clump/core mass function where the total number of objects is typically $\lesssim 100$. However, the CMF is easily misinterpreted, as we now demonstrate.

Consider a very simple mass function whose DMF is well-fit by a single power law of the form

$$
\frac{\Delta N}{\Delta M}=A M^{\alpha}
$$

where $\Delta N$ is the number of objects in a mass bin of width $\Delta M$, and $A$ and $\alpha$ are constants. 
It is sometimes assumed that the complementary cumulative mass function is also a power law whose exponent differs from $\alpha$ by 1 :

$$
\begin{aligned}
N(>M) & =\int_{M}^{\infty} \frac{\Delta N}{\Delta M} d M \\
& =-\frac{A}{\alpha+1} M^{\alpha+1},
\end{aligned}
$$

for $\alpha<-1$. The expectation is therefore that, if the DMF is well represented by a single power law, the CMF will be too. In measured mass functions, this may not be the case. A measured mass function contains a finite sample of clumps with an upper mass limit, $M_{\max }$. This upper mass limit can be either a real cutoff on the mass distribution or the result of finite sampling. In either case, the DMF will remain the simple power law of Equation 1, but the CMF will deviate from Equation 2. Integrating the DMF of Equation 2 to an upper limit of $M_{\max }$, we obtain

$$
\begin{aligned}
N(>M) & =\int_{M}^{M_{\max }} \frac{\Delta N}{\Delta M} d M \\
& =-\frac{A}{\alpha+1} M^{\alpha+1}+\frac{A}{\alpha+1} M_{\max }^{\alpha+1}
\end{aligned}
$$

The practical significance of the difference between Equations. 2 and 3 depends on the range of clump masses (i.e. the value of $M_{\max } / M_{\min }$ ), the value of the power-law exponent, $\alpha$, and the number of clumps in the sample, $\mathrm{N}_{\mathrm{cl}}$. In Figures 1 and 2, we use simulated data sets to show how the shape of both the DMF and CMF depend on each of these parameters. To simulate a clump mass function, we randomly draw $\mathrm{N}_{\mathrm{cl}}$ masses from the interval $M_{\min }$ to $M_{\max }$ following the distribution $\Delta N / \Delta M \propto M^{\alpha}$. We do this for representative values of $N_{\mathrm{cl}}=50,100$, and 1000. We simulate power law exponents of $\alpha=-1.5,-2.0$, and -2.5 . This range encompasses the exponents of the Salpeter IMF ( $\alpha=-2.35$; Salpeter 1955), the CO clump mass function ( $\alpha=-1.7$; Kramer et al. 1998), and the values typically found in submillimeter continuum clump surveys. We vary the ratio $M_{\max } / M_{\min }$ by holding $M_{\min }$ constant at 1 (the mass units are arbitrary) and taking $M_{\max }$ values of $10^{2}, 10^{3}, 10^{4}$, and $10^{5}$. For each combination of $\mathrm{N}_{\mathrm{cl}}, \alpha$, and $M_{\max }$, we produce a representative data set by averaging 1000 randomly-generated sets. Figures 1 and 2 show the resulting DMFs and CMFs, respectively. Note that we follow the popular convention of normalizing $N(>M)$ by $\mathrm{N}_{\mathrm{cl}}$. 


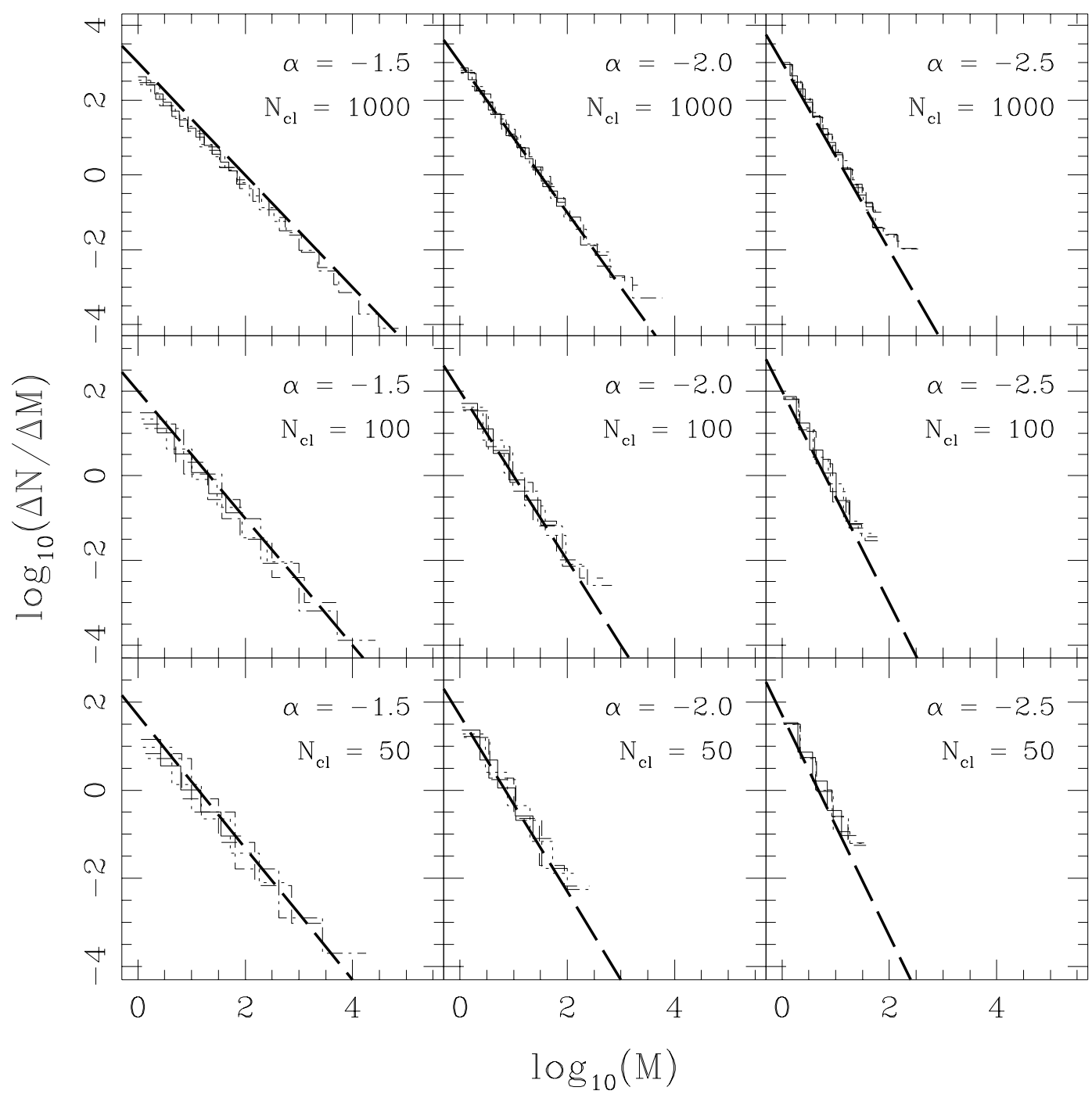

Fig. 1.- Differential mass functions from simulated clump mass distributions. Each simulated data set consists of $\mathrm{N}_{\mathrm{cl}}$ clumps drawn randomly from a mass function of the form $\Delta N / \Delta M \propto M^{\alpha}$ (thick dashed line), with $\mathrm{N}_{\mathrm{cl}}$ and $\alpha$ values as shown. To produce each plotted DMF, 1000 simulated data sets were averaged together. The four simulated DMFs plotted in each panel vary in the range of masses from which the simulated masses were drawn: 1-100 (solid line), 1-1000 (dotted line), 1-10 (short dashed line), and 1-10 (dotdashed line). The mass units are arbitrary. The number of bins in each mass function is set to $\left(2 \mathrm{~N}_{\mathrm{cl}}\right)^{1 / 3}$. 


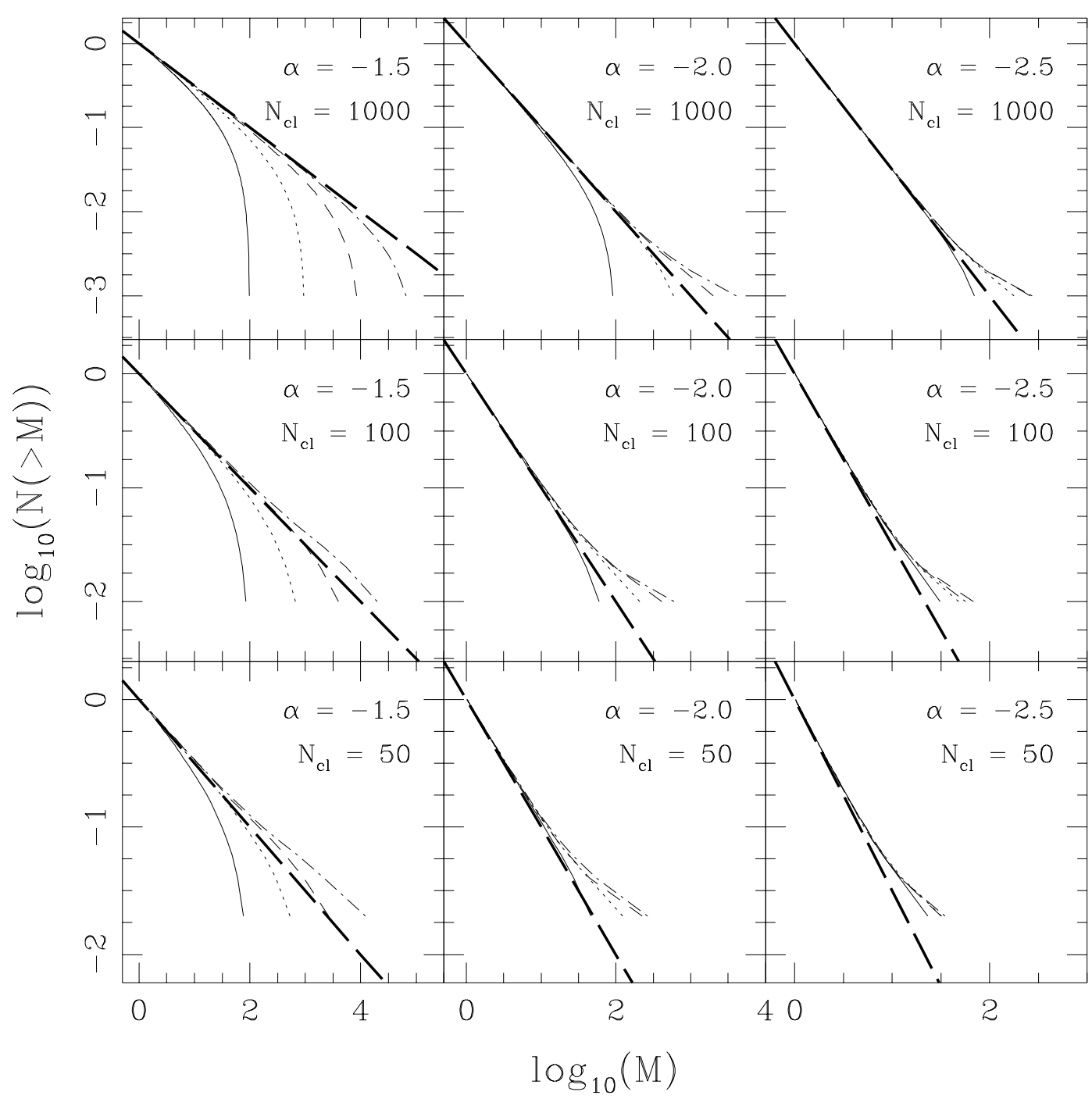

Fig. 2.- Cumulative mass functions from simulated clump mass distributions, corresponding to those whose differential mass functions are shown in Fig. 1. As in Fig. 1, the line types indicate the clump mass range from which simulated masses (arbitrary units) were drawn: 1-100 (solid line), 1-1000 (dotted line), 1-10 (short dashed line), and 1-10 (dot-dashed line). The thick dashed line represents the power law distribution from which the clump masses were drawn. For sufficiently steep power laws $(\alpha=-2.5)$, the CMF is likely to be well-fit by a single power law, even when the total number of clumps is small. However, for shallower power laws $(\alpha=-1.5)$ and CMFs spanning smaller clump mass ranges, the plotted CMF deviates significantly from the single power law form. 
By construction, the DMFs shown in Figure 1 all follow the input mass distributions. However, as shown in Figure 2, the CMFs clearly deviate from the power law form suggested by Equation 2. As seen in the right column of Figure 2, a steep power law $(\alpha=-2.5)$ overwhelms the effects of both $N_{\mathrm{cl}}$ and $M_{\mathrm{max}}$, recovering essentially the pure single power law in all cases. As seen in the middle and left columns of Figure 2, however, shallower power laws $(\alpha=-1.5,-2.0)$ often cause the CMF to deviate from the pure power law behaviour expected from Equation 2. This is especially the case when the ratio of $M_{\max } / M_{\min }$ is small (solid line in Fig. 2). It is therefore likely that fitting real data sets with single power laws of the form of Equation 2 would give highly misleading power law exponents. To achieve accurate results, fitted functions must account for the maximum clump mass, as in Equation 3.

Many of the simulated CMFs shown in Figure 2 strongly resemble the CMFs derived from observations of real star-forming regions. To account for their curvature, these observational CMFs are typically fitted with two power law segments (e.g. Johnstone et al. 2000). That intrinsically single power-law CMFs should so closely resemble those typically fit with two power laws implies that great care should be taken in interpreting the shape of the CMF and in choosing functions with which to fit it. In simple cases, such as the single power-law simulated here, a check of the DMF should help constrain the functional forms which might be fitted to the CMF. Even in this simple case, which is simpler than is likely to be encountered in real data, care must be taken when fitting the CMF in order to recover the correct power-law exponent: accounting for $M_{\max }$ is clearly essential. In CMFs

measured from real observations, the effects of incompleteness, small-number statistics, and binning can all lead to uncertainty about the functional form of the clump mass function. We believe that particular care is warranted where the observed clump/core mass function encompasses the peak in the stellar IMF at $\sim 0.1 M_{\odot}$ (Kroupa 2002; Chabrier 2003). In such cases, incompleteness and a real physical turnover in the mass function are likely to be difficult to distinguish.

\section{THE FUNCTIONAL FORM OF THE CLUMP MASS FUNCTION}

We now turn to the question of the functional form of the clump/core mass function. The form most commonly employed in studies of low-mass star-forming regions is a double power law above $\sim 0.1 M_{\odot}$ with a break between the power law segments at $\sim 0.5 M_{\odot}$ (Motte, André, \& Neri 1998; Johnstone et al. 2000; Motte et al. 2001; Johnstone et al. 2001). This is essentially the same form as found for the Galactic field single-star IMF (Kroupa 2002). However, a double power law is not the only plausible shape for the clump mass function. 
In Paper II, we showed that the M17 clump mass function is better fit by a lognormal distribution than by a double power law. Moreover, in $\S 2$ above, we noted that the finite clump mass range in any real clump mass function adds curvature to the CMF. This curvature can make a mass spectrum which is intrinsically a single power law appear to adopt a shape similar to those usually fit with a double power law. Additional uncertainties about the shape of the clump/core mass function arise due to the diversity of techniques used by different authors to fit such mass functions.

To eliminate the effects of differing fitting techniques, we have gathered all of the published lists of clump/core masses from unbiased millimeter and submillimeter continuum surveys of star-forming regions and fit them in a homogeneous manner. In Table 1, we summarize these 11 different clump/core mass functions measured in 7 different star-forming regions. In cases where the original authors used more than one set of assumptions about the dust temperature when calculating the clump masses, we use the masses calculated assuming a uniform dust temperature, which is the method most widely used. The only exceptions are the studies by Motte, André, \& Neri (1998) and Motte et al. (2001), which divide the clumps into several groups, each with a single dust temperature, before calculating their masses. Not all of the studies made comparable attempts to distinguish between starred and starless cores/clumps, so we have used the complete list of all objects in each case (except our own study of M17 from Paper II, where we have two comparable data sets in which clumps coincident with MSX sources were removed in a consistent way). The clumps in these 7 star-forming regions span more than 5 orders of magnitude in mass $\left(0.05-16000 M_{\odot}\right)$ and more than two orders of magnitude in linear size. The median clump masses range from $0.17 M_{\odot}$ in $\rho$ Oph to $470 M_{\odot}$ in RCW 106. For comparison with the theory of turbulent fragmentation, we also include in our analysis a list of clumps extracted from a computational hydrodynamic simulation of 4.6 Jeans masses of turbulent, self-gravitating gas (run B5 of Tilley \& Pudritz 2004, TP04 hereafter). There are two caveats to any comparison made between the TP04 and observational mass functions. First, the dimensionless nature of the TP04 simulations means the absolute mass scale is arbitrary. Second, the number of Jeans masses simulated by TP04 is considerably less than is found in real star-forming regions, which could have consequences for the number and mass distribution of fragments formed.

\subsection{Functional Forms Fitted}

In the literature, differential clump mass functions are usually fit by either a single power law, 
Table 1. Submillimeter and Millimeter Continuum Clump Surveys

\begin{tabular}{clccccccc}
\hline \hline Data Set ID & Region Name & $\lambda[\mu m]$ & $\mathrm{N}_{\text {clumps }}$ & HPBW $[\mathrm{pc}]$ & $\mathrm{M}_{\text {clump }}^{\text {median }}$ & $\mathrm{M}_{\text {clump }}^{\text {max }}$ & $\mathrm{M}_{\text {clump }}^{\text {tot }}$ & Ref. \\
\hline 1 & $\rho$ Oph & 1300 & 62 & 0.0085 & 0.17 & 3.2 & 24 & 1 \\
2 & $\rho$ Oph & 850 & 55 & 0.011 & 0.19 & 6.33 & 29 & 2 \\
3 & Orion B & 850 & 82 & 0.02 & 0.8 & 9.20 & 106 & 3 \\
4 & Orion B & 850 & 75 & 0.03 & 0.86 & 30.34 & 120 & 4 \\
5 & Lagoon/M8 & $450 \& 850$ & 37 & 0.13 & 6.93 & 34.1 & 287 & 5 \\
6 & M17 & 450 & 96 & 0.07 & 13 & 160 & 1700 & 6 \\
7 & M17 & 850 & 105 & 0.12 & 11 & 120 & 2100 & 6 \\
8 & NGC 7538 & 450 & 77 & 0.11 & 24 & 2700 & 8800 & 7 \\
9 & NGC 7538 & 850 & 67 & 0.21 & 34 & 3000 & 9300 & 7 \\
10 & W43 & 1300 & 48 & 0.29 & 135 & 3600 & $1.4 \times 10^{4}$ & 8 \\
11 & RCW 106 & 1200 & 95 & 0.42 & 470 & $1.6 \times 10^{4}$ & $9.9 \times 10^{4}$ & 9 \\
12 & simulation & $\ldots$ & 381 & $0.0014^{\mathrm{a}}$ & 0.045 & 25 & $57^{\mathrm{b}}$ & 10 \\
\hline
\end{tabular}

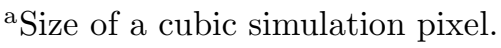

b Total mass of gravitationally bound clumps found in the simulation.

References. - (1) Motte, André, \& Neri (1998); (2) Johnstone et al. (2000); (3) Motte et al. (2001); (4) Johnstone et al. (2001); (5) Tothill et al. (2002); (6) Reid \& Wilson (2006); (7) Reid \& Wilson (2005); (8) Motte, Schilke, \& Lis (2003); (9) Mookerjea et al. (2004); (10) Tilley \& Pudritz (2004) 


$$
\frac{\Delta N}{\Delta M}=A M^{\alpha}
$$

or a double power law,

$$
\frac{\Delta N}{\Delta M}=\left\{\begin{aligned}
A M_{\mathrm{break}}^{\left(\alpha_{\mathrm{high}}-\alpha_{\mathrm{low}}\right)} M^{\alpha_{\mathrm{low}}} & , M<M_{\text {break }} \\
A M^{\alpha_{\mathrm{high}}} & , M \geq M_{\mathrm{break}}
\end{aligned}\right.
$$

where $\alpha_{\text {low }}$ and $\alpha_{\text {high }}$ are, respectively, the power-law exponents below and above the break mass, $M_{\text {break }}$. The curvature seen in observed clump CMFs makes it is clear that the single power law of Equation 4 cannot be applied to the CMF. However, many authors have made simple double power-law fits to the $\mathrm{CMF}$, as per

$$
N(>M)=\left\{\begin{aligned}
A M_{\text {break }}^{\left(\alpha_{\text {high }}-\alpha_{\text {low }}\right)} M^{\alpha_{\text {low }}+1} & , M<M_{\text {break }} \\
A M^{\alpha_{\text {high }}+1} & , M \geq M_{\text {break }}
\end{aligned}\right.
$$

Equation 6 is the first function with which we will fit observed CMFs, and we call it 2PL.

We can derive two other potential fitting functions by integrating Equations 4 and 5 to obtain their corresponding CMFs. As per the discussion of $\S 2$, we integrate to a finite upper mass limit, $M_{\max }$. The single power law CMF with an upper mass limit, which we call 1PLMM, is given by:

$$
N(>M)=\int_{M}^{M_{\max }} \frac{\Delta N}{\Delta M} d M=\left\{\begin{aligned}
\frac{A}{(\alpha+1)}\left(M_{\max }^{\alpha+1}-M^{\alpha+1}\right) & , M<M_{\max } \\
0 & , M \geq M_{\max }
\end{aligned}\right.
$$

Similarly the double power law CMF with an upper mass limit, which we call 2PLMM, is given by:

$$
N(>M)=\left\{\begin{aligned}
\frac{A M_{\mathrm{break}}^{\left(\alpha_{\mathrm{high}}-\alpha_{\mathrm{low}}\right)}}{\left(\alpha_{\mathrm{low}}+1\right)}\left(M_{\mathrm{break}}^{\alpha_{\mathrm{low}}+1}-M^{\alpha_{\mathrm{low}}+1}\right) & \\
+\frac{A}{\left(\alpha_{\mathrm{high}}+1\right)}\left(M_{\mathrm{max}}^{\alpha_{\mathrm{high}}+1}-M_{\mathrm{break}}^{\alpha_{\mathrm{high}}+1}\right) & , M<M_{\mathrm{break}} \\
\frac{A}{\left(\alpha_{\mathrm{high}}+1\right)}\left(M_{\mathrm{max}}^{\alpha_{\mathrm{high}}+1}-M^{\alpha_{\mathrm{high}}+1}\right) & , M_{\mathrm{break}} \leq M<M_{\mathrm{max}} \\
0 & , M \geq M_{\max } .
\end{aligned}\right.
$$

Inspired by similar work on the stellar mass function, we also consider a lognormal DMF: 


$$
\frac{\Delta N}{\Delta M}=\frac{1}{A_{1} \sqrt{2 \pi} M} \exp \left[-\frac{\left(\ln M-A_{0}\right)^{2}}{2 A_{1}^{2}}\right],
$$

whose complementary CMF, which we call LOGN, is given by:

$$
N(>M)=\frac{1}{2}\left[1-\operatorname{erf}\left(\frac{\ln M-A_{0}}{\sqrt{2} A_{1}}\right)\right] .
$$

We constructed a CMF for each region using the published masses. To assign weights to

each point for use in the calculation and minimization of $\chi^{2}$, we make the same assumptions as in Paper II, namely that the mass uncertainties, $\sigma_{M}$, are distributed roughly as $\sigma_{M} \propto M$, and that the CMF can be approximately represented by a power law. Under these assumptions, the uncertainties in $N(>M), \sigma_{N}$, are approximately given by $\sigma_{N} \propto N$ (see Paper II). This method of assigning weights avoids the use of individual mass uncertainties, which are frequently unavailable. To assess the uncertainty in each fitted parameter, we must again choose a method which does not rely on the mass uncertainties. For each CMF, we construct and fit $10^{5}$ Monte Carlo realizations of the data using the bootstrap method (random sampling with replacement, e.g. Press et al. 1992). Using the resulting distributions of fitted parameters, which are typically symmetric about the best-fit values, we construct 95\% ( $\sim 2 \sigma)$ confidence limits on each parameter and report these as our uncertainties. Using the distribution of $\chi^{2}$ values, we compute the probability, $P$, of obtaining by chance a $\chi^{2}$ value poorer than that from the best fit to the original CMF. Higher $P$ values indicate a better quality of fit.

Figures 3-6 show all eleven measured CMFs with the best fit versions of these functions: 2PL, 1PLMM, 2PLMM, and LOGN. The CMF of the simulated clumps from TP04 is included for comparison. The $P$ values for all of the fits are summarized in Table 2.

\subsection{Fits to Observed Clump Mass Functions}

Figures 3-6 and the $\mathrm{P}$ values shown in Table 2 indicate that none of the observational CMFs is best fit by a single power law, 7 are best fit by some form of the double power law (either 2PL or 2PLMM), and 4 are best fit by a lognormal clump mass distribution (LOGN). Before discussing each case in turn, we encourage the reader to keep in mind that small differences in the $P$ values between two almost equally good fits are probably not meaningful. In calculating the $P$ values, we had to use the bootstrap approximation in place of the frequently-unavailable clump mass uncertainties, and this makes the $P$ values themselves uncertain. 


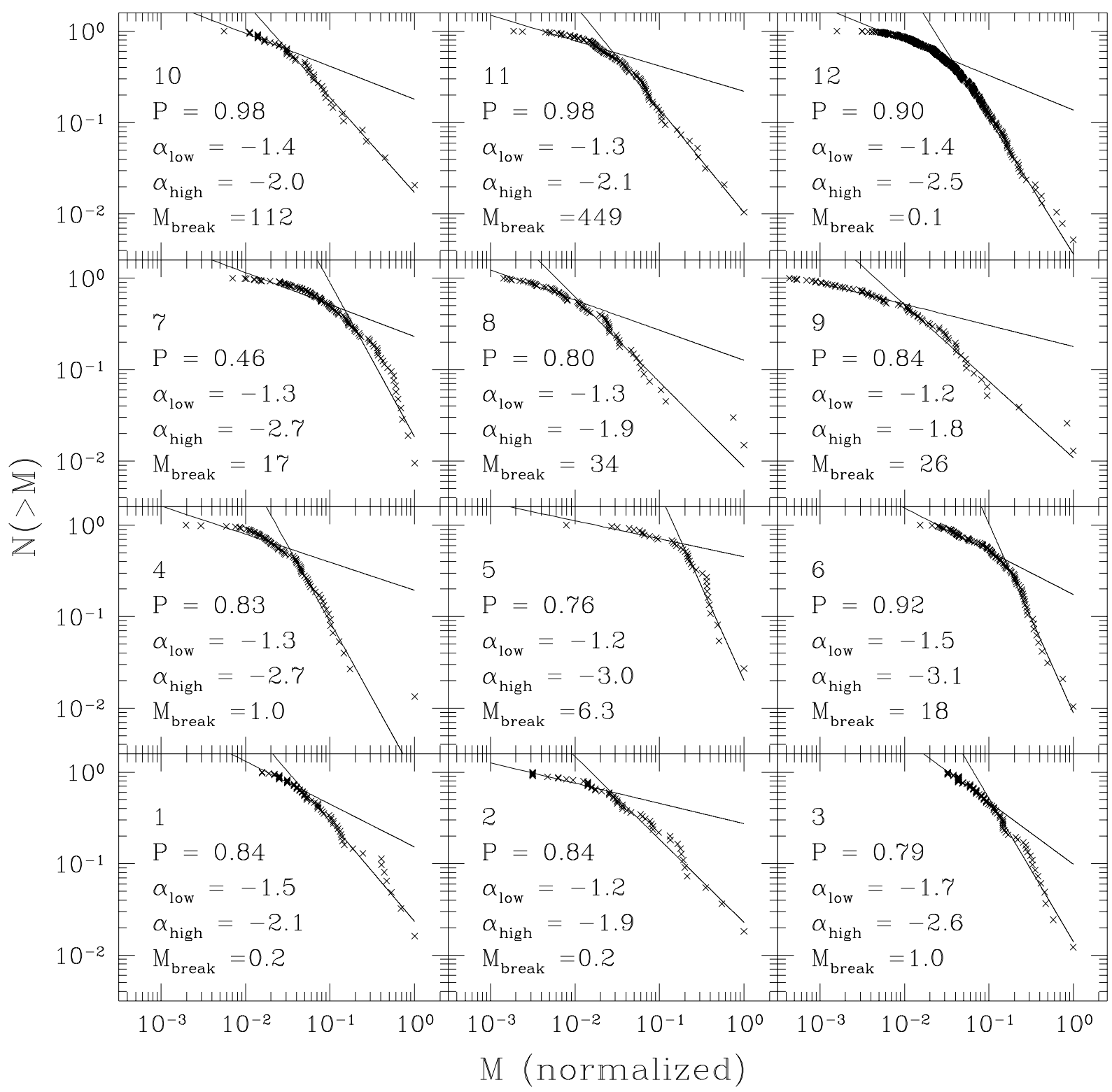

Fig. 3.-Cumulative mass functions (symbols) for the 12 data sets described in Table 1. Each CMF is fit by a pure double power law (2PL), as described by Equation 6. The panel labels indicate the data set ID from Table 1, goodness-of-fit probability, $P$, and best-fit parameters, $\alpha_{\text {low }}, \alpha_{\text {high }}$, and $M_{\text {break }}$. For visual clarity, the maximum clump mass in each plot has been normalized to unity; the best fit parameters given are for fits to the unnormalized data. 


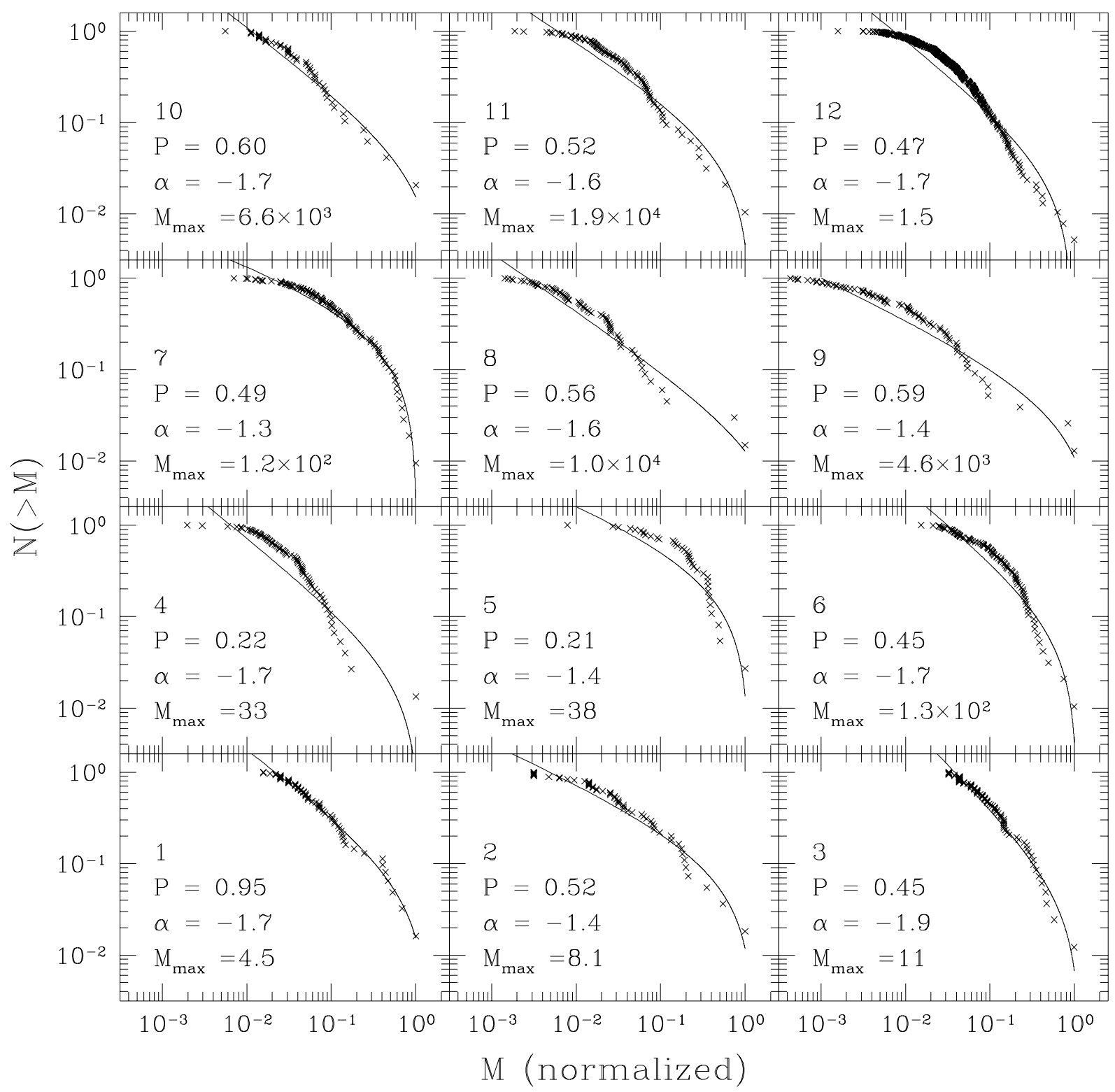

Fig. 4.- Cumulative mass functions (symbols) for the 12 data sets described in Table 1. Each CMF is fit by a single power law (1PLMM) described by Equation 7. The panel labels indicate the data set ID from Table 1, goodness-of-fit probability, $P$, and best-fit parameters, $\alpha$ and $M_{\max }$. For visual clarity, the maximum clump mass in each plot has been normalized to unity; the best fit parameters given are for fits to the unnormalized data. 


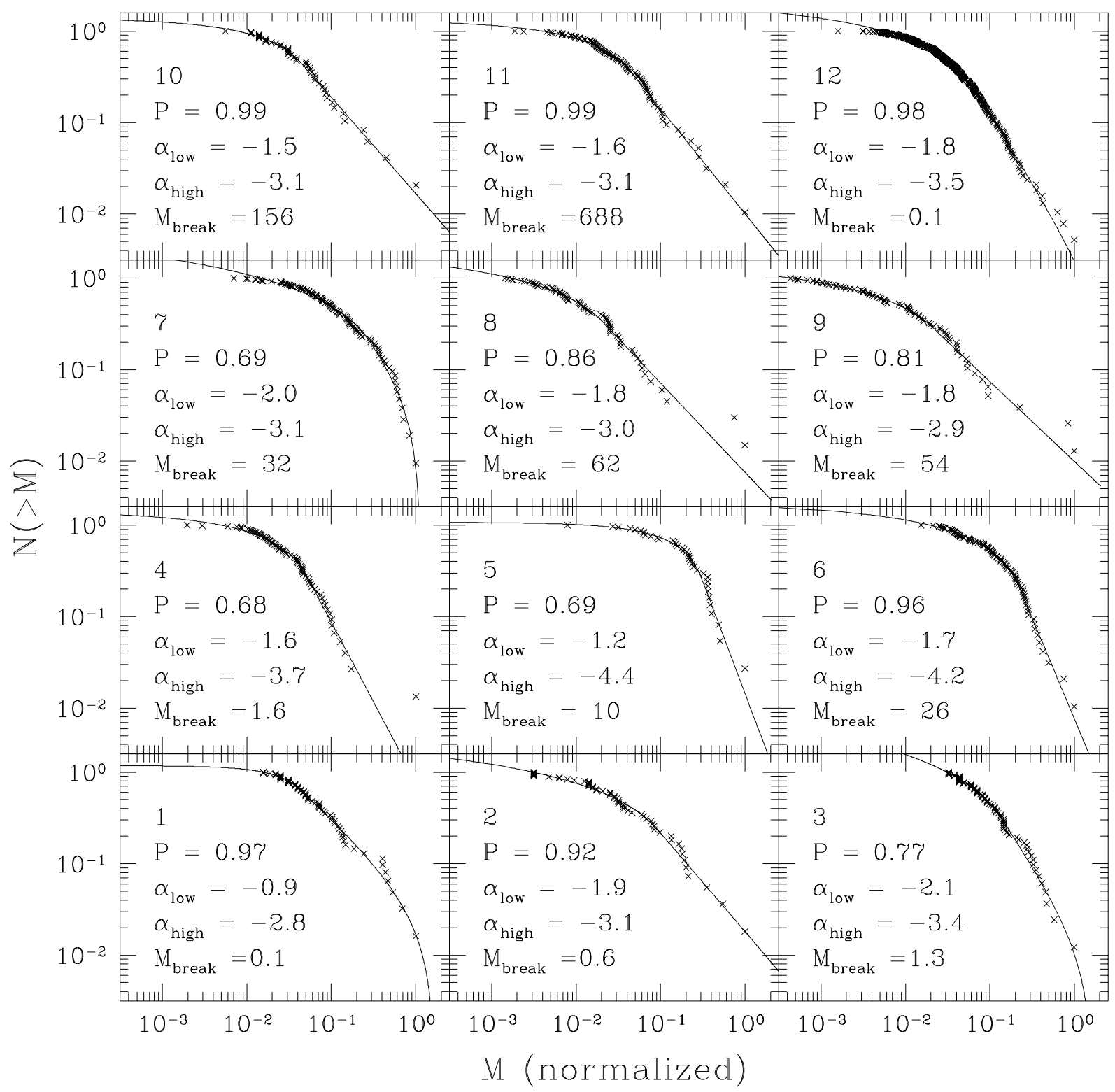

Fig. 5.- Cumulative mass functions (symbols) for the 12 data sets described in Table 1. Each CMF is fit by the double power law (2PLMM) described by Equation 8. The panel labels indicate the data set ID from Table 1, goodness-of-fit probability, $P$, and best-fit parameters, $\alpha$ and $M_{\max }$. For visual clarity, the maximum clump mass in each plot has been normalized to unity; the best fit parameters given are for fits to the unnormalized data. 


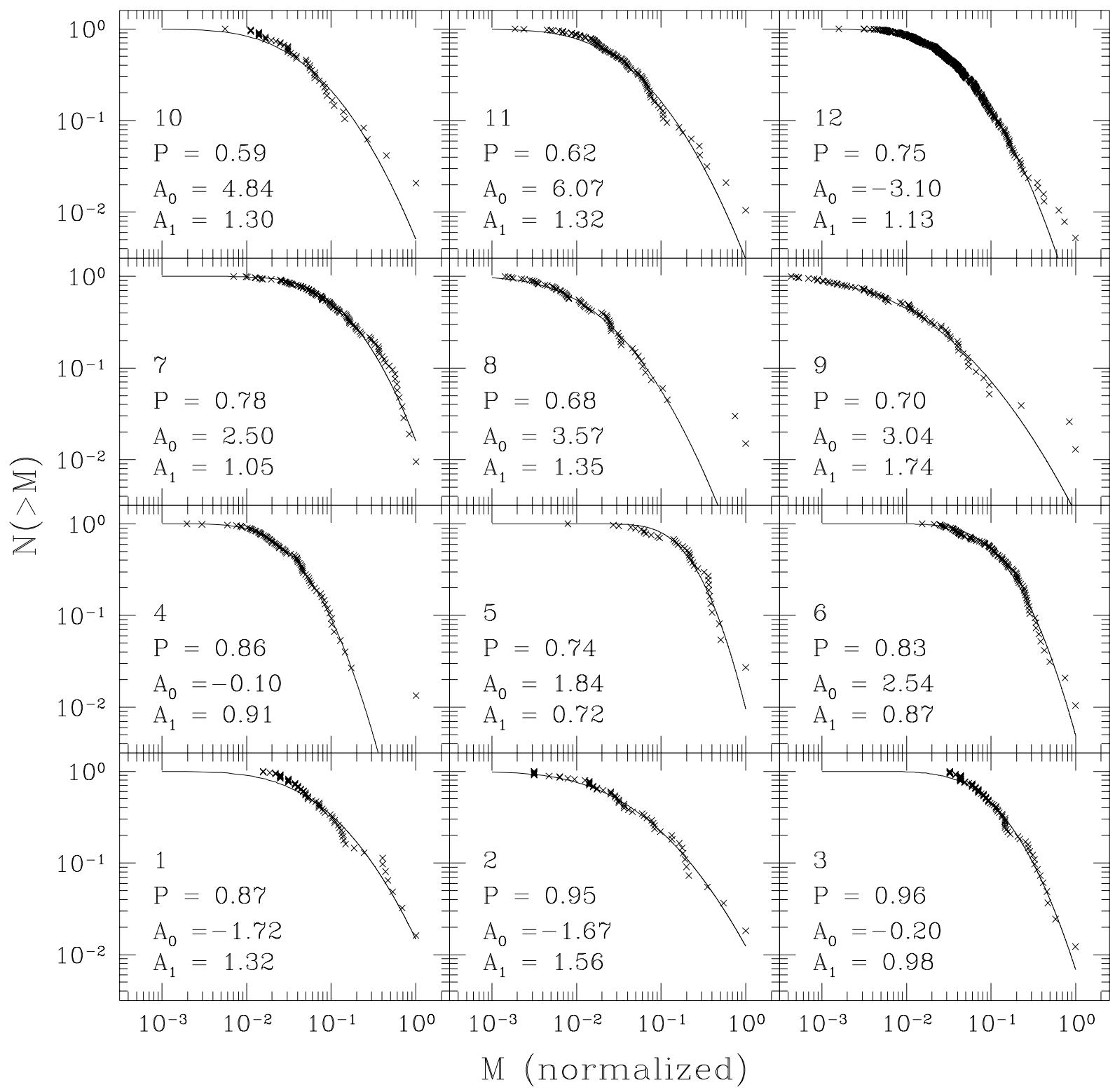

Fig. 6.-Cumulative mass functions (symbols) for the 12 data sets described in Table 1. Each CMF is fit by a lognormal clump mass distribution (LOGN), as described by Equation 10. The panel labels indicate the data set ID from Table 1, goodness-of-fit probability, $P$, and best-fit parameters, $A_{0}$ and $A_{1}$. For visual clarity, the maximum clump mass in each plot has been normalized to unity; the best fit parameters given are for fits to the unnormalized data. 
Table 2. $\quad P$ Values for Fits to CMFs

\begin{tabular}{lccccc}
\hline \hline & & \multicolumn{4}{c}{ Fit Type } \\
\cline { 3 - 5 } \multicolumn{1}{c}{ Region Name } & Data Set ID & 2PL & 1PLMM & 2PLMM & LOGN \\
\hline$\rho$ Oph $(1.3 \mathrm{~mm})$ & 1 & 0.84 & 0.95 & $\mathbf{0 . 9 7}$ & 0.87 \\
$\rho$ Oph $(850 \mu \mathrm{m})$ & 2 & 0.84 & 0.52 & 0.92 & $\mathbf{0 . 9 5}$ \\
Orion B $(850 \mu \mathrm{m})$ & 3 & 0.79 & 0.45 & 0.77 & $\mathbf{0 . 9 6}$ \\
Orion B $(850 \mu \mathrm{m})$ & 4 & 0.83 & 0.22 & 0.68 & $\mathbf{0 . 8 6}$ \\
Lagoon & 5 & $\mathbf{0 . 7 6}$ & 0.21 & 0.69 & 0.74 \\
M17 $(450 \mu \mathrm{m})$ & 6 & 0.92 & 0.45 & $\mathbf{0 . 9 6}$ & 0.83 \\
M17 $(850 \mu \mathrm{m})$ & 7 & 0.46 & 0.49 & 0.69 & $\mathbf{0 . 7 8}$ \\
NGC $7538(450 \mu \mathrm{m})$ & 8 & 0.80 & 0.56 & $\mathbf{0 . 8 6}$ & 0.68 \\
NGC $7538(850 \mu \mathrm{m})$ & 9 & $\mathbf{0 . 8 4}$ & 0.59 & 0.81 & 0.70 \\
W43 & 10 & 0.98 & 0.60 & $\mathbf{0 . 9 9}$ & 0.59 \\
RCW 106 & 11 & 0.98 & 0.52 & $\mathbf{0 . 9 9}$ & 0.62 \\
Run B5 of TP04 & 12 & 0.90 & 0.47 & $\mathbf{0 . 9 8}$ & 0.75 \\
\hline
\end{tabular}

Note. - Fit types are: direct double power-law fit to the CMF (2PL), single power law DMF integrated to a finite upper mass limit (1PLMM), double power law DMF integrated to a finite upper mass limit (2PLMM), and a lognormal DMF (LOGN). The highest $P$ value for each star-forming region is displayed in bold text. 
$\boldsymbol{\rho}$ Oph - The Motte, André, \& Neri (1998) $1.3 \mathrm{~mm}$ core CMF (data set 1) is best fit by the 2 PLMM distribution, although the 1PLMM fit is only marginally poorer. Similarly, the Johnstone et al. (2000) $850 \mu \mathrm{m}$ core CMF (set 2) is best fit by the LOGN distribution, but the $2 \mathrm{PLMM}$ fit is only marginally poorer. Given that the two data sets represent the same clump sample, we expect that the same function should describe them. Either the 2PLMM or LOGN functions could serve this purpose, but the 2PLMM function gives a slightly higher mean $P$ value. Interestingly, the LOGN fit, which has only 2 parameters, nearly matches the quality of the 2PLMM function, which has 5 .

Orion B - In this region the lognormal mass function provides the best fit to both measurements of the CMF. The $2 \mathrm{PL}$ function provides an almost equally good fit to data set 4 , but not to data set 3 . Visual inspection of Figures 3 and 6 affirms the superiority of the LOGN fit. If we exclude the massive outlier clump at the high-mass end of set 4 , the $P$ value for the LOGN fit rises to 0.99 and that for the $2 \mathrm{PL}$ fit falls to 0.78 . We suspect that the massive outlier is probably a composite clump which would resolve into multiple less-massive objects if observed at higher angular resolution.

Lagoon - Although Tothill et al. (2002) argued for a single power law fit to this mass function (set 5 ), the $P$ values indicate that the $2 \mathrm{PL}$ function provides a significantly better fit $(P=0.76$ versus $P=0.21)$. The lognormal fit, with $P=0.74$, is only marginally poorer than the 2PL fit. Note that the study from which these data were obtained is unique in having used a by-eye clump extraction method.

M17 - As in Paper II, we find that the $450 \mu \mathrm{m} \mathrm{CMF} \mathrm{(set} \mathrm{6)} \mathrm{is} \mathrm{better} \mathrm{fit} \mathrm{by} \mathrm{a} \mathrm{double}$ power law (2PLMM, in this case), while the $850 \mu \mathrm{m} \mathrm{CMF} \mathrm{(set} \mathrm{7)} \mathrm{is} \mathrm{better} \mathrm{fit} \mathrm{by} \mathrm{a} \mathrm{lognormal}$ distribution. Both the LOGN and 2PLMM functions might be said to provide credible fits to both data sets, though again the LOGN function does this using three fewer parameters than does the 2PLMM function.

NGC 7538 - In this case, the $P$ values show that some form of double power law (2PLMM for set 8 and 2PL for set 9) provides the best fit to both measurements of the CMF. The three most massive clumps in each CMF correspond to the IRS 1-3, IRS 9, and IRS 11 regions. Each of these regions is already known to be forming more than one star and therefore likely contains significant unresolved structure. For example, the IRS 1-3 region contains 3 compact Hir regions (Israel 1977; Wynn-Williams, Becklin, \& Neugebauer 1974). If we omit these outliers from the analysis, the $P$ values for the lognormal fits become 0.97 and 0.69 for data sets 8 and 9 , respectively. The corresponding $P$ values for the 2PLMM fits become 0.99 and 0.96. Therefore, we conclude that, although the LOGN function remains a contender, the NGC 7538 CMF is probably best fit by a double power law. 
W43 and RCW 106 - These two massive star-forming regions provide the clearest cases of CMFs (sets 10 \& 11) which are best fit by double power laws. In both cases, the 2PLMM fits are favored, with $P=0.99$, while the $2 \mathrm{PL}$ fits both give $P=0.98$. No other function provides a comparably good fit.

Run B5 of TP04 - Here again, the 2PLMM function provides the best fit, with $P=0.99$. Visual inspection of the plot suggests that the LOGN function fits equally well, although the $P$ values suggest otherwise.

To summarize, the general trend in the fits is toward lognormal mass functions among the low-mass star-forming regions and some form of the double power law mass function among the massive star-forming regions. The two major sources of uncertainty in this analysis are the choice of weights and the use of the bootstrap technique. Both would be mitigated if the original clump mass uncertainties were available. If we use uniform weights in making the fits, instead of the default weights of $1 / N^{2}$, the LOGN function then provides the best fit in 5 cases, the 2PLMM in 4 , and they tie in 3 . With regard to the use of the bootstrap technique, we note that although using the real clump mass uncertainties would be preferable, the $P$ values obtained herein using the bootstrap technique show the same trend as those obtained in Paper II using the proper uncertainties. We strongly encourage the reporting of both random and systematic clump mass uncertainties in future studies.

A potential fault with the 2PLMM fits can be seen by comparing the best fit parameters from the 2PL fits (Fig. 3) with those from the 2PLMM fits (Fig. 5). The 2PL $\alpha_{\text {high values }}$ range around the Salpeter value of $\alpha=2.35$ but the mean 2PLMM value is $\left\langle\alpha_{\text {high,2PLMM }}\right\rangle=$ $\left\langle\alpha_{\text {high,2PL }}\right\rangle-1.0$. Moreover, the mean ratio of the break masses derived from the 2PLMM and 2PL fits is 1.6. The 2PLMM fits are achieving the excellent $P$ values they do by shifting the break mass up and fitting the top end of the mass function with a very steep power law - one which is not obviously related to the power laws which fit the CO clump or stellar mass functions, both of which are significantly shallower. It is also important to note that the 2PLMM fits achieve their excellent $P$ values using 5 adjustable parameters, compared to 4 for the $2 \mathrm{PL}$ fits and only 2 for the LOGN fits. For the remainder of the discussion in this paper, we will refer primarily to the power law exponents derived from the 2PL fits, which are most suitable for direct comparison to those obtained by other authors.

\subsection{Interpreting the Fits}

Previous studies of the core mass function in regions of low-mass star formation have emphasized the agreement between the shapes of the core and stellar mass functions (e.g. 
Testi \& Sargent 1998; Motte, André, \& Neri 1998; Johnstone et al. 2000), when both are fit with similar numbers of power laws over similar mass ranges. The core mass functions in $\rho$ Oph, Orion B, and Serpens are all well fit by a Salpeter-like power law above about $1 M_{\odot}$ $\left(\alpha_{\text {high }} \simeq-2.35\right)$, flatten out somewhat below about $1 M_{\odot}\left(\alpha_{\text {low }} \simeq 1.5\right)$, and peak at about $0.1 M_{\odot}$.

In Table 3, we summarize the best-fit parameters for the 2PL and LOGN fits to the 12 CMFs studied herein. Fitting all of the CMFs in the same way, we find $\alpha_{\text {high }}$ values ranging between -1.8 and -3.1 , comparable to the range of power law exponents found in fits to the $\gtrsim 1 M_{\odot}$ stellar IMF (Kroupa 2002). The mean of the $\alpha_{\text {high }}$ values is $-2.4 \pm 0.1$, or $-2.3 \pm 0.2$ if we include only the 7 observational CMFs which are best fit by double power laws. Both measures of the mean $\alpha_{\text {high }}$ are compatible with the Salpeter IMF, despite the differences in the clump mass ranges over which the various $\alpha_{\text {high }}$ values were derived. Similarly, if the low-mass ends of all 11 observational CMFs are fit with power laws, the resulting exponents, $\alpha_{\text {low }}$, range between -1.2 and -1.7 , with a mean of $-1.4 \pm 0.1$. This result also accords with the shallowing of the stellar IMF at its low-mass end, despite the differences in the clump mass scales over which this shallowing occurs. Perhaps most interesting is that, as shown in Figure 7 , we find no trend in the $\alpha_{\text {high }}$ values with the median clump mass in each region. This result applies over nearly four orders of magnitude in median clump mass. The power law indices derived from fits to the mass functions of submillimeter continuum clumps/cores offer no clear way to distinguish between low-mass regions, such as $\rho$ Oph, and very high-mass regions, such as RCW 106. The median clump mass in RCW 106 is nearly $500 M_{\odot}$, which suggests that most of the clumps are probably cluster-forming objects, yet the RCW 106 clump mass function and the $\rho$ Oph core mass function may be fit with compatible power laws.

We emphasize four key results from this fitting exercise. First, there is the tendency toward lognormal CMFs in less massive star-forming regions and power-law CMFs in massive star-forming regions. Second, there is the agreement among the $\alpha_{\text {high }}$ and $\alpha_{\text {low }}$ values from region to region, despite the significant differences in the relevant clump mass scales. Third, there is the presence of a break between the steep and shallow sections of the mass function in high-mass star-forming regions. If this break is real, it implies that the clump mass function is not a single unbroken power law above $1 M_{\odot}$. This result holds true despite the uncertainties in the absolute mass calibration in each study (see §3.3.4) Finally, there is the agreement between the power law exponents of the clump and stellar mass functions, again despite the differences in mass scales. 


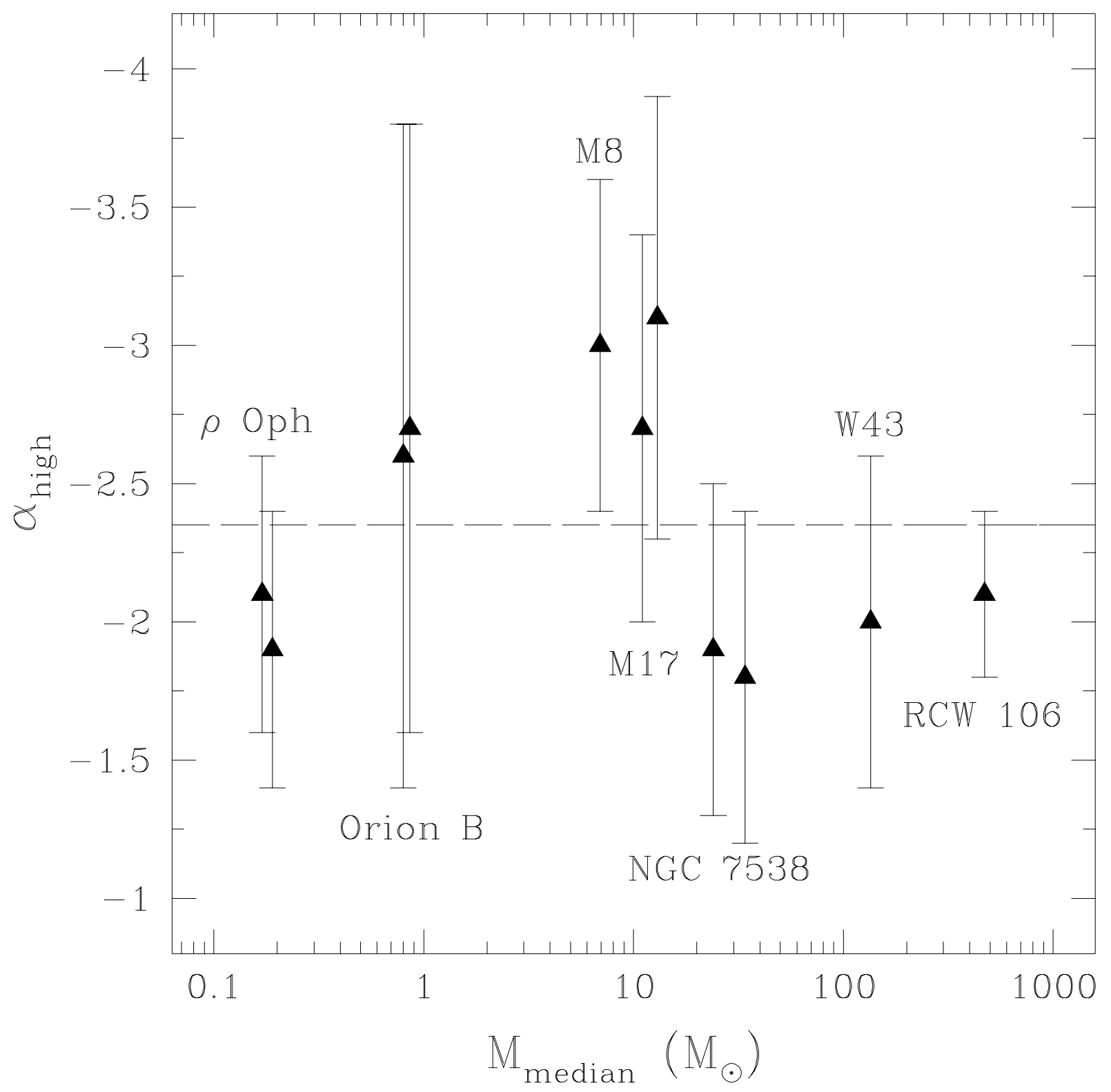

Fig. 7.- Power-law exponent, $\alpha_{\text {high }}$, versus median clump mass for the 11 millimeter/submillimeter clump mass functions described in Table 1 . The $\alpha_{\text {high values are derived }}$ from the pure double power law fits (2PL, Eq. 6). The horizontal dashed line indicates the Salpeter exponent, $\alpha_{\text {high }}=-2.35$ (Salpeter 1955). The error bars represent the $95 \%(\sim 2 \sigma)$ confidence limits from Table 3 . No trend in $\alpha_{\text {high }}$ with mass is apparent. 


\subsubsection{Lognormal vs. Double Power-Law CMFs}

There is already ample theoretical evidence that lognormal mass functions can result when any sufficiently stochastic process, such as turbulent fragmentation, governs the evolution of the clump masses. Moreover, lognormal mass functions can be viewed as one extremum of a continuum of shapes whose other extremum is the power-law mass function. Numerical simulations have repeatedly shown that turbulent fragmentation can naturally produce both power-law and lognormal mass functions whose shapes are largely independent of the intrinsic mass scale (Klessen, Burkert, \& Bate 1998; Klessen \& Burkert 2000; Klessen 2001; Padoan \& Nordlund 2002; Gammie et al. 2003; Tilley \& Pudritz 2004; Clark \& Bonnell 2006). Several authors have invoked the Central Limit Theorem to demonstrate analytically that lognormal mass functions arise whenever a large number of random parameters act to set the clump masses (Larson 1973; Zinnecker 1984; Adams \& Fatuzzo 1996). Conversely, power-law mass functions can arise when fewer random parameters affect each clump's mass. These random parameters could derive from successive turbulent fragmentations or N-body gravitational encounters among clumps. We might therefore imagine the following scenario to explain the trend toward lognormal mass functions in low-mass regions and power-law mass functions in high-mass regions (see Table 2). A molecular cloud undergoes turbulent fragmentation, producing an initially power-law clump mass function; as the clumps continue to fragment and interact, their mass function approaches a lognormal distribution; by the time the "clumps" have become "cores", they have achieved a fully lognormal mass distribution. As the objects furthest down the chains of turbulent fragmentation and Nbody interactions, the masses of the cores would have been maximally influenced by random processes.

In this scenario, spatially unresolved observations of a set of cores whose mass distribution is lognormal could effectively restore the less-evolved double power law mass function by blurring together smaller cores into semblances of their antecedent clumps. This hypothesis would explain the trend toward double power law mass functions seen in the more distant regions, such as NGC 7538, W43, and RCW 106, where the spatial resolution of the observations isn't sufficient to adequately distinguish all of the cores from one another.

\subsubsection{The Clump CMF vs. the Stellar IMF}

If the core/clump mass function in both low- and high-mass star-forming regions were found to be consistent with the stellar IMF (uncorrected for unresolved multiples), it would relax the need to invoke processes such as coalescence and competitive accretion (Bonnell et al. 1997, 2001; Bonnell, Vine, \& Bate 2004) to explain the formation of more massive stars. 
Such consistency would also argue in favor of theories which predict that massive stars can form by a scaled-up version of molecular cloud core collapse with disk accretion (McKee \& Tan 2002, 2003; Krumholz, McKee, \& Klein 2005; Krumholz 2006).

As shown in Table 3, the mean power law exponents from the double power law fits to the 11 observational CMFs are $\alpha_{\text {high }}=-2.4 \pm 0.1$ and $\alpha_{\text {low }}=-1.4 \pm 0.1$. Kroupa (2002) cites power law exponents for the mean Galactic field single-star IMF of $-2.3 \pm 0.3$ for $M_{\text {star }} / M_{\odot} \geq 0.5$ and $-1.3 \pm 0.5$ for $0.08 \leq M_{\text {star }} / M_{\odot}<0.5$. The agreement between the $\alpha_{\text {high }}$ values of the clump and stellar mass functions is intriguing. Unlike the $\alpha_{\text {low }}$ values, which may be strongly affected by incompleteness in the clump mass functions (see next

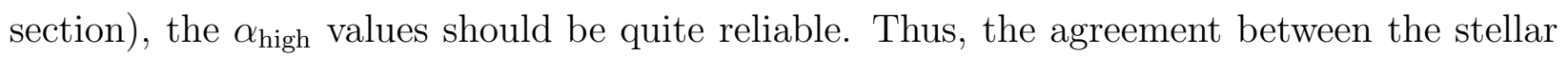
and clump $\alpha_{\text {high }}$ values reinforces the ideas that the stellar IMF originates in the mass function of clumps in a fragmented molecular cloud and that the stellar mass function can be obtained by an essentially one-to-one conversion of clumps into stars (or binaries). If the high-mass end of the stellar IMF can be obtained by converting clumps into stars on a more-or-less one-to-one basis, there may be no need to invoke processes such as coalescence or competitive accretion.

Kroupa (2002) has shown that the variation in the measured power law exponents for the stellar IMF is compatible with normal measurement variation and need not represent physical region-to-region variation. We suggest that the same description could apply equally well to the range of power law exponents derived from clump mass functions in different regions (see Fig. 7). More measurements of $\alpha_{\text {high }}$ would be required to confirm this hypothesis in the cases of both the stellar IMF and the clump mass function. Elmegreen (2004) has shown that real region-to-region variations in the IMF are physically plausible, but may be masked by small-number statistics, which is certainly a concern with the clump/core mass functions taken to date.

Can we identify a mass scale at which the apparent agreement between the clump and stellar mass functions breaks down? Of the parameters which control the shapes of the fitted functions, namely $A_{1}$ for the lognormal fits and the $\alpha$ values for the power law fits, none shows a trend with the median clump mass of the regions (see Table 3). Thus, the fits suggest that the shape of the clump mass function is not very sensitive to the clump mass range being fit. There is also no clear break between the CMFs which do mirror the shape of the stellar IMF and those which do not, despite the breadth in the clump mass ranges. Again, this surprising result suggests that the intrinsic shape of the stellar IMF may be apparent in the clump mass function on mass and linear scales substantially larger than those previously considered. 


\subsubsection{Incompleteness and Spatial Filtering}

Both interferometry and the chopping technique used by single-dish submillimeter telescopes filter out emission on some spatial scales. Clump-finding algorithms may have a similar effect. Therefore, all of the observations used to generate the CMFs discussed in this paper are subject to some form of spatial filtering, and hence to incompleteness and uncertainty. We are therefore motivated to ask whether spatial filtering and related effects introduced during data acquisition and reduction can introduce a sufficient number of random perturbations to the clump masses to ensure that a lognormal mass function is obtained.

Spatial filtering of flux can systematically alter the derived clump masses. The magnitude of the discrepancy would depend on the size (and therefore the mass) of each clump. Although this possibility should be explored further, several arguments suggest it does not substantially alter the shape of the clump mass function. First, the mass function of clumps extracted from infrared dust extinction maps, which are essentially free of spatial filtering, is very similar to those derived from submillimeter continuum observations (J. Alves, private communication). If the clump extraction algorithms exerted a strong spatial filtering effect, we would need to understand how at least three different methods used by the authors of the papers studied here all produce similar mass functions. Moreover, the effects of spatial filtering would affect observations of both low- and high-mass star-forming regions. If it were a significant effect, it seems unlikely that observations of low-mass star-forming regions would still manage to produce a core mass function which appears to agree with the stellar IMF. Finally, we note that the clump CMF extracted from the turbulent fragmentation simulations of TP04 is not subject to observational spatial filtering and its shape is entirely compatible with those of the observational CMFs (TP04 used a custom clump extraction technique). For these reasons, it is difficult to explain how the shape of the clump mass function, and its similarity from region to region might be accounted for by spatial filtering.

Whether or not the clump mass functions are strongly affected by spatial filtering, they are certainly all affected by incompleteness. For spatially extended objects, the detection threshold is a limiting surface brightness, meaning that a clump of any mass could go undetected if its flux were spread thinly across the sky. Thus, the clump sample may be incomplete in any mass range. At the moment, there is no reliable way to correct millimeter and submillimeter continuum clump/core mass functions for incompleteness. If incompleteness is a significant determinant of the shape of each CMF, its effects are likely to be felt most strongly at their low-mass ends, where it may be partly responsible for the shallower slope of the mass function. Consequently, the degree of incompleteness might influence the

position of the break, $M_{\text {break }}$, between the two power laws when a double power law is fitted 
to a given mass function. In most studies to date, the distribution of clumps in mass-radius space shows a (sometimes slight) separation from the detection threshold at its high-mass end (Reid \& Wilson 2005, 2006; Motte et al. 2001). Hence, we do not think it likely that the fitted $\alpha_{\text {high }}$ values are strongly affected by sensitivity-induced incompleteness. The $\alpha_{\text {low }}$ values, however, are likely to be significantly impacted by incompleteness.

There are several ways to test for the related effects of incompleteness and spatial filtering. High spatial resolution observations of the more distant star-forming regions in the sample will reveal the extent to which currently unresolvable structure will change the shape of the clump/core mass function. Observations of more distant regions should also mitigate the effects of chopping by limiting the loss of emission to spatial scales much larger than those of typical molecular cloud cores. Comparison of submillimeter continuum and dust extinction maps of identical regions should provide strong constraints on the effect of spatial filtering in the former type of observations. Finally, observations with total power detectors, such as SCUBA-2, will eliminate the effects of chop-based spatial filtering. SCUBA-2's greater sensitivity will also help reveal those clumps not detectable by the present generation of submillimeter and millimeter detectors.

\subsubsection{Uncertainties in Absolute Mass Calibration}

It should be noted that, while there are significant uncertainties in the absolute calibration of clump masses, these uncertainties are not large enough to fundamentally change the conclusions of the preceding analysis. The calibration of the absolute mass scale in each study we reference relies on assumptions about the dust temperature and emissivity in each region. Typically, as in Papers I and II, values typical of each region are applied to all of the clumps it contains. The dust emissivity in star-forming regions may be uncertain by up to a factor of 2 (i.e. it may range between $\sim 1-2$ ) and the temperature may be uncertain by perhaps a factor of four (i.e. the mean clump temperature is likely to lie within 10-40 K). In the most extreme case, whereby the mean temperature and dust emissivity in a region are assumed to be $10 \mathrm{~K}$ and 1, respectively, but are actually $40 \mathrm{~K}$ and 2 , the clump masses would all be overestimated by a factor of $\sim 30$. However, referring to Table 3 , we see that even this large uncertainty is insufficient to account for the observed variation in the peak mass, $M_{\text {peak }}$ from the LOGN fits or the break mass, $M_{\text {break }}$, from the 2 PL fits. Both of these parameters range over $>3$ orders of magnitude, which is a much greater variation than can be accounted for by the largest plausible uncertainties in the dust emissivity and temperature. In addition, the mass calibrations are unlikely to be incorrect by the same amount or in the same direction in all of the studies. Finally, we emphasize that uncertainties in the 
absolute mass calibration do not affect the shapes of the various clump mass functions.

\section{REGION-TO-REGION VARIATIONS IN THE CLUMP MASS FUNCTION}

The clump mass function is one of the most important descriptors of the initial conditions for star formation. It tells us how the masses of pre-stellar condensations are distributed, leaving us to determine how such a distribution could give rise to the observed distribution of stellar masses. Thus, it is important to understand how the clump mass function varies from region to region, between low- and high-mass star-forming regions, and whether it varies more or less than the stellar IMF. This information will help evaluate theories which posit different formation mechanisms for stars of different masses. For example, if low- and high-mass star forming regions had systematically different clump mass functions but compatible stellar mass functions, it would be evidence that the mechanisms of low- and high-mass stars were different.

In Figure 8, we plot all 11 of the observational CMFs from Table 1 in the same panel. To facilitate comparisions among the mass functions, they have all been renormalized such that they share a common median clump mass. This renormalization preserves the shape of each mass function. Like Figure 7, Figure 8 shows that there is no clear trend in the shape of the mass function with the median mass in each region. Particularly at their high-mass ends, where incompleteness is least significant, there is no clear segregation between the CMFs of low- and high-mass star-forming regions.

In the previous section, we described how parameters derived from fits to the clump mass function vary from one star-forming region to another. However, to more precisely quantify the extent to which the shape of the clump mass function varies from region to region, it is best to use non-parametric techniques. Such techniques do not rely on prior knowledge of the functional form of the clump mass function. One such test is the Kolmogorov-Smirnov (K-S) test, which measures the probability , $P$, that two sets of clump masses represent random samplings from the same parent distribution. We compared each of the mass functions listed in Table 1 to the others using the K-S test. To establish a baseline for interpreting the K-S test $P$ values, we compare similar sets of mass functions. In Figure 9, we plot pairs of independent measurements of the $\mathrm{CMF}$ in each of the four regions where multiple measurements are available ( $\rho$ Oph, Orion B, NGC 7538, and M17). The $P$ values for these comparisons range from 0.72 for $\rho$ Oph to 0.96 for Orion B. The lowest $P$ value results from comparing studies (Motte, André, \& Neri 1998; Johnstone et al. 2000) of $\rho$ Oph which differ in their authors, continuum wavebands, clump extraction techniques, and assumptions 


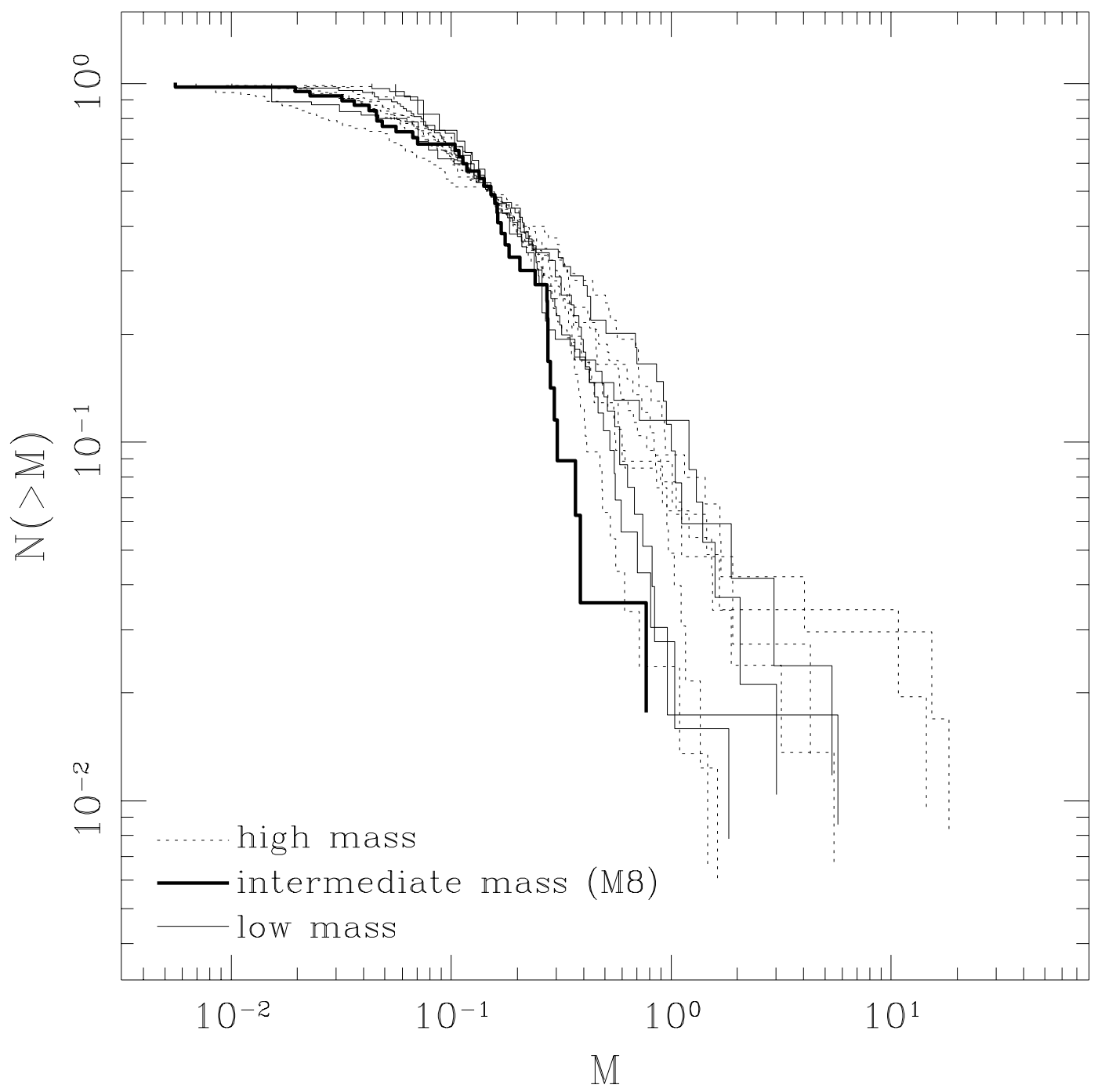

Fig. 8.- Cumulative clump mass functions for all 11 observational studies listed in Table 1. The masses of the clumps in each CMF have been rescaled such that all clump sets have a common median mass. CMFs of low-mass star-forming regions ( $\rho$ Oph and Orion B) are plotted with solid lines, that of M8 with a thick solid line, and those of the massive starforming regions (M17, NGC 7538, W43, and RCW 106) with dotted lines. The new mass scale is arbitrary. 
about the clump temperatures. The two intermediate $P$ values come from our own studies of NGC 7538 (Paper I) and M17 (Paper II). Each pair differs only by the continuum waveband of the observations. The highest $P$ value derives from the comparison of the two Orion B studies (Motte et al. 2001; Johnstone et al. 2001), and they differ by authors, clump extraction techniques, and assumptions about temperatures, but not in continuum waveband. In principle, each pair of mass functions should be the nearly identical, as they represent different measures of essentially the same thing. Thus, the magnitude of the difference between the two should primarily reflect differences introduced during data reduction and analysis. Hence, we set our threshold for statistically significant similarity between two CMFs at $P=0.72$, the lowest measured probability for comparisons of similar data sets. Note that these results agree well with those from the previous section: we find the highest K-S $P$ value when comparing the two Orion B studies, which were both found to be well-fit by lognormal distributions in $\S 3$, and the lowest K-S $P$ value when comparing the $\rho$ Oph mass functions, which were found to be best-fit by different functions.

Table 4 shows the $P$ values for pair-wise K-S test comparisons of all of the mass functions described in Table 1. Clearly, a direct K-S comparison of regions such as $\rho$ Oph and RCW 106 would yield a $P$ value of 0 because their clump mass ranges do not overlap. To enable a comparison of the shapes of any two mass functions regardless of their intrinsic mass scales, we renormalize each pair of mass functions so they share a common median clump mass, as we did for all of the CMFs in Figure 8. In Figure 10 we plot the pair-wise comparisons of a representative set of 6 clump mass functions along with the $P$ value for each comparison. The $P$ values for the 15 unique comparisons range from 0.73 to 1.00, implying that, in every case, when the mass functions are set on a common mass scale, there is a significant likelihood that they represent random samples from the same clump mass distribution. Examining the $P$ values for all 55 unique inter-comparisons between the 11 observational CMFs (Table 4), we find that only 11 fall below the threshold of statistical significance $(P=0.72)$, though none is lower than $P=0.56$. Of these 11,4 are associated with the Motte et al. (2001) study of Orion B, which differs from the rest in having large numbers of duplicate clump masses. Long runs of identical masses open gaps between the Motte et al. (2001) CMF and the others. The K-S test interprets these gaps as statistically significant discrepancies when, in fact, they may only reflect the number of significant figures in the reported clump masses. This effect may account for the lower average $P$ values for comparisons involving the Motte et al. (2001) Orion B CMF, compared to those involving the very similar study of Orion B by Johnstone et al. (2001), whose $P$ values indicate statistically significant similarity in every case.

Thus, without reference to the functional form of the clump/core mass function, we find that, when set on a common mass scale, all of the CMFs have a statistically significant 
Table 3. Best Fit Parameters for 2PL and LOGN Fits

\begin{tabular}{|c|c|c|c|c|c|c|c|c|}
\hline \multirow[b]{2}{*}{ Region } & \multirow{2}{*}{$\begin{array}{c}\text { Data } \\
\text { Set ID }\end{array}$} & \multicolumn{4}{|c|}{ LOGN Fit Parameters } & \multicolumn{3}{|c|}{ 2PL Fit Parameters } \\
\hline & & $\overline{A_{0}}$ & $\overline{A_{1}}$ & $M_{\text {peak }}{ }^{\mathrm{a}}$ & $\langle M\rangle^{\mathrm{a}}$ & $M_{\text {break }}$ & $\alpha_{\text {low }}$ & $\alpha_{\text {high }}$ \\
\hline$\rho \mathrm{Oph}(1.3 \mathrm{~mm})$ & 1 & $-1.7 \pm 0.3$ & $1.3 \pm 0.3$ & $0.03 \pm 0.01$ & $0.43 \pm 0.09$ & $0.2 \pm 0.2$ & $-1.5 \pm 0.3$ & $-2.1 \pm 0.5$ \\
\hline$\rho \mathrm{Oph}(850 \mu \mathrm{m})$ & 2 & $-1.7 \pm 0.4$ & $1.6 \pm 0.4$ & $0.016 \pm 0.008$ & $0.6 \pm 0.2$ & $0.2 \pm 0.1$ & $-1.2 \pm 0.1$ & $-1.9 \pm 0.5$ \\
\hline Orion $\mathrm{B}(850 \mu \mathrm{m})$ & 3 & $-0.2 \pm 0.2$ & $1.0 \pm 0.2$ & $0.31 \pm 0.05$ & $1.3 \pm 0.2$ & $1 \pm 1$ & $-1.7 \pm 0.2$ & $-2.6 \pm 1.2$ \\
\hline Orion $\mathrm{B}(850 \mu \mathrm{m})$ & 4 & $-0.1 \pm 0.2$ & $0.9 \pm 0.2$ & $0.39 \pm 0.07$ & $1.4 \pm 0.2$ & $1.0 \pm 0.6$ & $-1.3 \pm 0.2$ & $-2.7 \pm 1.1$ \\
\hline Lagoon & 5 & $1.8 \pm 0.3$ & $0.7 \pm 0.3$ & $3.7 \pm 0.8$ & $8 \pm 1$ & $6 \pm 2$ & $-1.2 \pm 0.2$ & $-3.0 \pm 0.6$ \\
\hline $\operatorname{M17}(450 \mu \mathrm{m})$ & 6 & $2.5 \pm 0.2$ & $0.9 \pm 0.2$ & $6 \pm 1$ & $18 \pm 2$ & $18 \pm 8$ & $-1.5 \pm 0.1$ & $-3.1 \pm 0.8$ \\
\hline M17 $(850 \mu \mathrm{m})$ & 7 & $2.5 \pm 0.3$ & $1.0 \pm 0.1$ & $4.1 \pm 0.7$ & $21 \pm 3$ & $17 \pm 10$ & $-1.3 \pm 0.1$ & $-2.7 \pm 0.7$ \\
\hline NGC $7538(450 \mu \mathrm{m})$ & 8 & $3.6 \pm 0.3$ & $1.3 \pm 0.6$ & $6 \pm 3$ & $90 \pm 30$ & $30 \pm 30$ & $-1.3 \pm 0.2$ & $-1.9 \pm 0.6$ \\
\hline NGC $7538(850 \mu \mathrm{m})$ & 9 & $3.0 \pm 0.4$ & $1.7 \pm 0.6$ & $1.0 \pm 0.7$ & $90 \pm 40$ & $30 \pm 30$ & $-1.2 \pm 0.1$ & $-1.8 \pm 0.6$ \\
\hline W43 & 10 & $4.8 \pm 0.3$ & $1.3 \pm 0.5$ & $20 \pm 10$ & $300 \pm 90$ & $110 \pm 80$ & $-1.4 \pm 0.2$ & $-2.0 \pm 0.6$ \\
\hline RCW 106 & 11 & $6.1 \pm 0.2$ & $1.3 \pm 0.4$ & $80 \pm 30$ & $1000 \pm 200$ & $400 \pm 300$ & $-1.3 \pm 0.2$ & $-2.1 \pm 0.3$ \\
\hline Run B5 of TP04 & 12 & $-3.1 \pm 0.1$ & $1.1 \pm 0.1$ & $0.013 \pm 0.002$ & $0.085 \pm 0.007$ & $0.06 \pm 0.02$ & $-1.4 \pm 0.1$ & $-2.5 \pm 0.3$ \\
\hline Mean values $^{b}$ & & & $1.2 \pm 0.1$ & & & & $-1.4 \pm 0.1$ & $-2.4 \pm 0.1$ \\
\hline
\end{tabular}

Note. - All quoted uncertainties correspond to a $95 \%(\sim 2 \sigma)$ confidence interval.

aThe peak mass, $M_{\text {peak }}$, and mean mass, $\langle M\rangle$, of the lognormal distribution are not parameters of the fit; they are calculated from $A_{0}$ and $A_{1}$ using the equations $\langle M\rangle=\exp \left(A_{0}+\frac{1}{2} A_{1}^{2}\right)$ and $M_{\text {peak }}=\exp \left(A_{0}-A_{1}^{2}\right)$.

${ }^{\mathrm{b}}$ Mean values include the 11 observational CMFs but exclude the CMF from the simulations of TP04.

Table 4. Kolmogorov-Smirnov $P$ Values for Mass Function Comparisons

\begin{tabular}{|c|c|c|c|c|c|c|c|c|c|c|c|c|}
\hline Region Name & Data Set ID & 2 & 3 & 4 & 5 & 6 & 7 & 8 & 9 & 10 & 11 & 12 \\
\hline$\rho \operatorname{Oph}(1.3 \mathrm{~mm})$ & 1 & 0.72 & 0.87 & 0.95 & 0.82 & 0.63 & 0.80 & 0.83 & 0.60 & 0.73 & 0.81 & 0.68 \\
\hline$\rho \mathrm{Oph}(850 \mu \mathrm{m})$ & 2 & $\ldots$ & 0.77 & 0.95 & 0.65 & 0.81 & 1.00 & 0.98 & 0.77 & 0.90 & 0.96 & 0.96 \\
\hline Orion $\mathrm{B}(850 \mu \mathrm{m})$ & 3 & $\ldots$ & $\ldots$ & 0.96 & 0.67 & 0.68 & 0.64 & 0.83 & 0.60 & 0.76 & 0.77 & 0.69 \\
\hline Orion B $(850 \mu \mathrm{m})$ & 4 & $\cdots$ & $\cdots$ & $\cdots$ & 0.92 & 0.91 & 0.94 & 0.87 & 0.79 & 1.00 & 0.99 & 0.98 \\
\hline Lagoon & 5 & $\ldots$ & $\ldots$ & $\ldots$ & $\cdots$ & 0.90 & 0.70 & 0.56 & 0.72 & 0.91 & 0.64 & 0.70 \\
\hline $\operatorname{M17}(450 \mu \mathrm{m})$ & 6 & $\cdots$ & $\cdots$ & $\ldots$ & $\cdots$ & $\cdots$ & 0.77 & 0.88 & 0.82 & 0.98 & 0.99 & 0.81 \\
\hline $\operatorname{M17}(850 \mu \mathrm{m})$ & 7 & $\cdots$ & $\cdots$ & $\cdots$ & $\cdots$ & $\cdots$ & $\cdots$ & 0.91 & 0.78 & 0.96 & 0.99 & 0.99 \\
\hline NGC $7538(450 \mu \mathrm{m})$ & 8 & $\cdots$ & $\cdots$ & $\cdots$ & $\ldots$ & $\cdots$ & $\cdots$ & $\cdots$ & 0.84 & 0.97 & 0.99 & 0.95 \\
\hline NGC $7538(850 \mu \mathrm{m})$ & 9 & $\cdots$ & $\ldots$ & $\cdots$ & $\ldots$ & $\ldots$ & $\cdots$ & $\cdots$ & $\ldots$ & 0.71 & 0.87 & 0.81 \\
\hline W43 & 10 & $\ldots$ & $\ldots$ & $\ldots$ & $\ldots$ & $\ldots$ & $\ldots$ & $\ldots$ & $\ldots$ & $\ldots$ & 0.98 & 0.97 \\
\hline RCW 106 & 11 & $\ldots$ & $\cdots$ & $\cdots$ & $\cdots$ & $\cdots$ & $\cdots$ & $\cdots$ & $\cdots$ & $\cdots$ & $\cdots$ & 0.98 \\
\hline
\end{tabular}

Note. - Bold text indicates those values which fall below the threshold of statistical significance $(P=0.72$; see text $)$. 


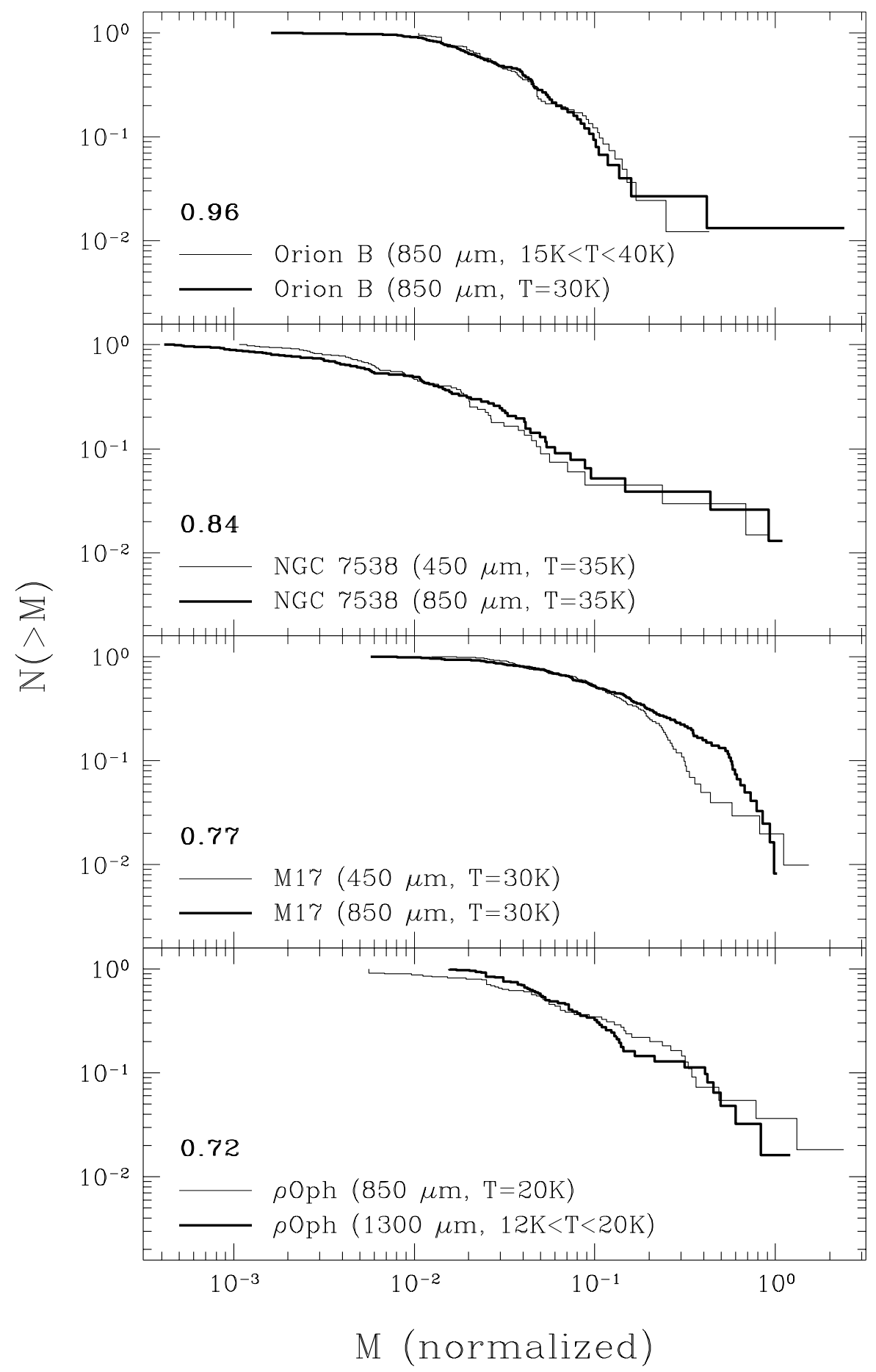

Fig. 9.- Comparison of the cumulative clump mass functions in four different star-forming regions, using data at different wavelengths and/or drawn from different studies. In each panel, the bold number indicates the K-S test $P$ value for the comparison of the two CMFs. 


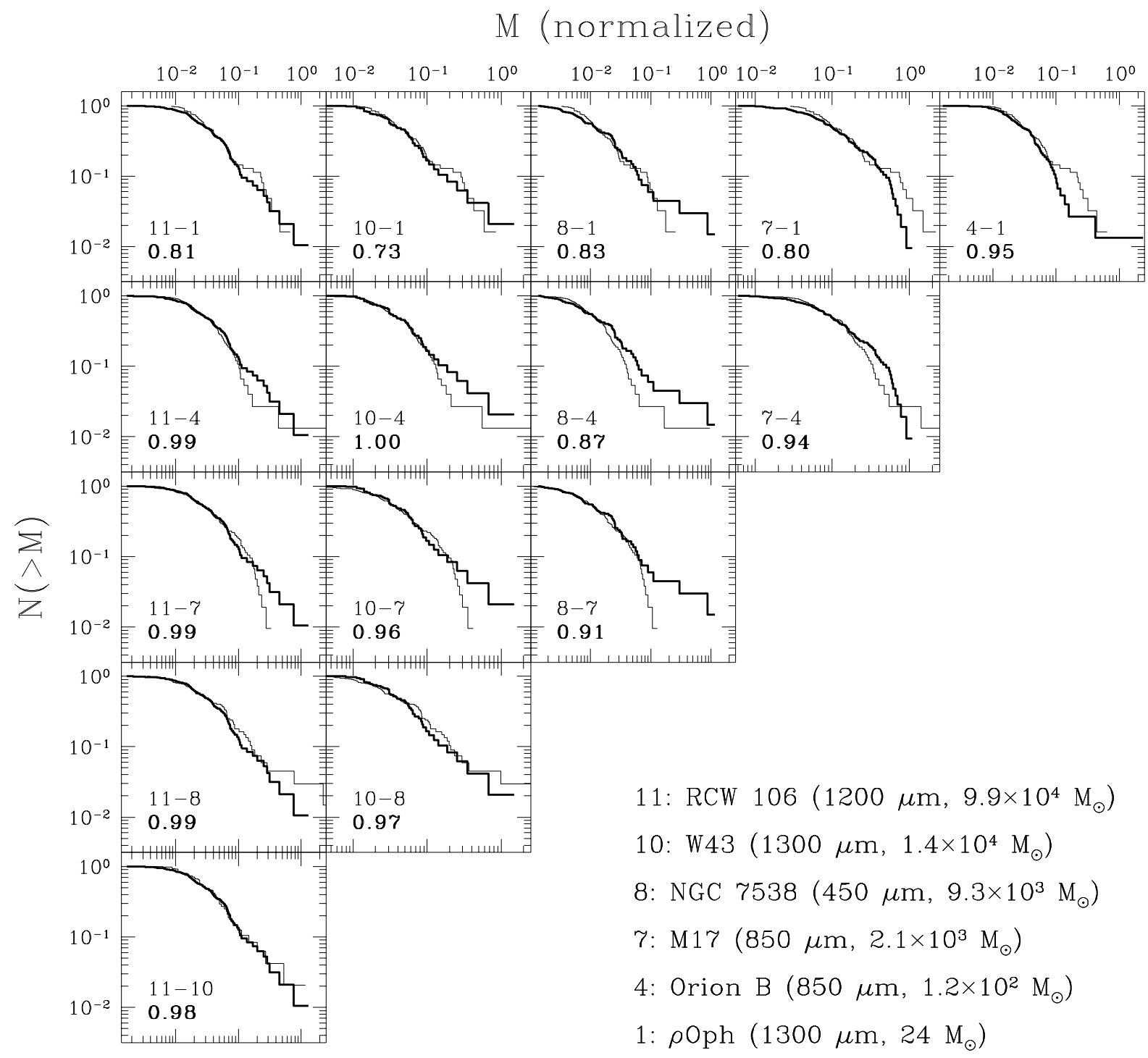

Fig. 10.- Comparison of the cumulative mass functions (CMFs) of six different star-forming regions, spanning more than five orders of magnitude in clump mass $\left(0.05\right.$ to $\left.\sim 16000 \mathrm{M}_{\odot}\right)$. In the lower left corner of each panel, the regions being compared are identified by their ID numbers, $x-y$, from Table 1 . The most massive clump in CMF $x$ (thick line) is normalized to mass unity and CMF $y$ (thin line) is normalized accordingly (see text). The boldface number in each panel is the Kolmogorov-Smirnov $P$ value for the comparison (see text for interpretation). For each region, the wavelength at which the continuum observations were made and the total mass of clumps detected are given in the legend. 
likelihood of representing random samples drawn from the same parent distribution. In other words, the CMFs all appear to have the same (or very similar) shapes, despite the differences in their intrinsic mass scales. Because the K-S test is least sensitive to the high- and low-mass tails of the CMFs, it is less affected by the few most massive clumps in each region which, as we saw in $\S 3$, can bias the determination of the functional form of the CMF. The combined results of the parametric fits from $\S 3$ with the non-parametric K-S test comparisons provide compelling evidence that the shapes of the clump and core mass distributions in all of the regions considered are strongly linked to each other and perhaps also to the stellar IMF.

\section{SUMMARY}

In this paper, we have examined the issue of the functional form of the clump/core mass function derived from millimeter/submillimeter continuum observations of Galactic star-forming regions. We have demonstrated that, even in the case of a strict single powerlaw mass function, great care must be taken in fitting the cumulative form of the mass function to accurately measure the exponent of this power law. Unexpected curvature can be introduced into the CMF when the range of masses or the number of objects is small, particularly when the power law is shallow $\left(\Delta N / \Delta M \propto M^{\alpha}\right.$ with $\left.\alpha \gtrsim-2\right)$. In some cases, an intrinsically single power-law mass function might appear better fit by two or more power laws. In practice, the mass function is unlikely to be a pure single power law, so the potential to be mislead when fitting the cumulative mass function is even greater.

We have fit 11 independent measurements of the clump/core mass function extracted from millimeter and submillimeter continuum observations of 7 star-forming regions. The masses of the clumps/cores in these regions collectively span more than five orders of magnitude. We find that, in regions where the median clump/core mass is less than a few $M_{\odot}$, the clump/core mass function is best fit by a lognormal distribution. In more massive regions, a double power law provides a better fit to the clump CMF. At intermediate masses, both the lognormal and the double power law adequately describe the CMF. This difference in the functional form of the clump mass function may represent an evolution toward a lognormal mass function at lower masses and smaller spatial scales. This interpretation is consistent with theoretical explanations for the origin of a lognormal mass function via stochastic processes such as turbulent fragmentation and gravitational N-body interactions.

The shape of the clump/core mass function does not appear to be a strong function of the intrinsic mass scale. The transition from lognormal to double power-law mass functions is fairly subtle. The mean value of the power-law exponent of the high-mass end of the clump/core mass function is found to be $\alpha_{\text {high }}=-2.4 \pm 0.1$, consistent with the Salpeter 
mass function. This result suggests that the shape of the stellar IMF may be apparent in the clump mass function on clump mass scales of several tens of $M_{\odot}$ or more and linear scales of several tenths of a parsec. It also suggests that molecular cloud cores may be converted into stars (or small multiple systems) on an essentially one-to-one basis. This scenario is consistent with theories in which massive stars form by core collapse and disk accretion. It also relaxes the need to invoke either coalescence or competitive accretion to explain the formation of massive stars, though these processes probably do occur at some level. This result deserves further investigation, especially to constrain any instrumental or post-processing effects which may affect it.

The similarities among the shapes of the various clump/core mass functions are confirmed to be statistically significant using non-parametric pair-wise comparisons of the CMFs. In 44 of 55 comparisons, after normalizing away the differences in their median masses, the clump/core mass functions are found to have a high likelihood of representing random samplings from the same parent distribution. That the observational CMFs agree well with the $\mathrm{CMF}$ of clumps extracted from a simulation of self-gravitating turbulent gas suggests that turbulent fragmentation is one possible sufficient explanation for the shape of the clump/core mass function.

M. A. R. has been supported by an Ontario Graduate Scholarship in Science and Tech-

nology. Both M. A. R. and C. D. W. are supported by the Natural Sciences and Engineering Research Council of Canada (NSERC). M. A. R. would like to thank E. Feigelson and F. Motte for helpful discussions during the preparation of this manuscript.

\section{REFERENCES}

Adams, F. C., \& Fatuzzo, M. 1996, ApJ, 464, 256

Bonnell, I. A., Bate, M. R., Clarke, C. J., \& Pringle, J. E. 1997, MNRAS, 285, 201

Bonnell, I. A., Clarke, C. J., Bate, M. R., \& Pringle, J. E. 2001, MNRAS, 324, 573

Bonnell, I. A., Larson, R. B., \& Zinnecker, H. 2006, preprint(astro-ph/0603447)

Bonnell, I. A., Vine, S. G., \& Bate, M. R. 2004, MNRAS, 349, 735

Chabrier, G. 2003, PASP, 115, 763

Clark, P. C. \& Bonnell, I. A. preprint (astro-ph/0603578) 
Elmegreen, B. G. 2004, MNRAS, 354, 367

Gammie, C. F., Lin, Y., Stone, J. M., \& Ostriker, E. C. 2003, ApJ, 592, 203

Israel, F. P. 1977, A\&A, 59, 27

Johnstone, D. J., Wilson, C. D., Moriarty-Schieven, G., Joncas, G., Smith, G., Gregersen, E., \& Fich, M. 2000, ApJ, 545, 327

Johnstone, D. J., Fich, M., Mitchell, G. F., Moriarty-Schieven, G. 2001, ApJ, 559, 307

Klessen, R. S., Burkert, A., \& Bate. M. R. 1998, ApJ, 501, L205

Klessen, R. S. 2001, ApJ, 556, 837

Klessen, R., \& Burkert, A. 2000, ApJS, 128, 287

Kramer, C., Stutzki, J., Röhrig, R., \& Corneliussen, U. 1998, A\&A, 329, 249

Kroupa, P. 2002, Science, 295, 82

Krumholz, M. R., McKee, C. F., \& Klein, R. I. 2005, ApJ, 618, 33

Krumholz, M. R. 2006, ApJ, 641, L45

Larson, R .B. 1973, MNRAS, 161, 133

Maíz-Apellániz, J. \& Úbeda L. 2005, preprint (astro-ph/0505012)

McKee, C. F., \& Tan, J. C. 2002, Nature, 416, 6876

McKee, C. F., \& Tan, J. C. 2003, ApJ, 585, 2

Mookerjea, B., Kramer, C., Nielbock, M., \& Nyman, L.-A. 2004, A\&A, 426, 119

Motte, F., André, P., Ward-Thompson, D., Bontemps, S. 2001, A\&A, 372, L41

Motte, F., André, P., \& Neri, R. 1998, A\&A, 336, 150

Motte, F., Schilke, P., \& Lis, D.C. 2003, ApJ, 582, 277

Padoan, P. \& Nordlund, Å. 2002, ApJ, 576, 870

Press, W. H., Teukolsky, S. A., Vetterling, W. T., \& Flannery, B. P. 1992, Numerical Recipes in Fortran, (2nd ed., Cambridge: University Press)

Reid, M. A., \& Wilson, C. D. 2005, ApJ, 625, 891 
Reid, M. A., \& Wilson, C. D. 2005, ApJ, submitted

Salpeter, E. E. 1955, ApJ, 121, 161

Scalo, J. 1998, in ASP Conf. Ser. 142, The Stellar Initial Mass Function Proceedings, ed. G. Gilmore \& D. Howell (San Francisco: ASP), 201

Testi, L. \& Sargent, A. I. 1998, ApJ, 508, L91

Tilley, D. A. \& Pudritz, R. E. 2004, MNRAS, 353, 769

Tothill, N. F. H., White, G. J., Matthews, H. E., McCutcheon, W. H., McCaughrean, M. J., Kenworthy, M. A. 2002, ApJ, 580, 285

Williams, J. P., de Geus, E. J., \& Blitz, L. 1994, ApJ, 428, 693

Wynn-Williams, C. G., Becklin, E. E., \& Neugebauer, G. 1974, ApJ, 187, 473

Zinnecker, H. 1984, MNRAS, 210, 43 